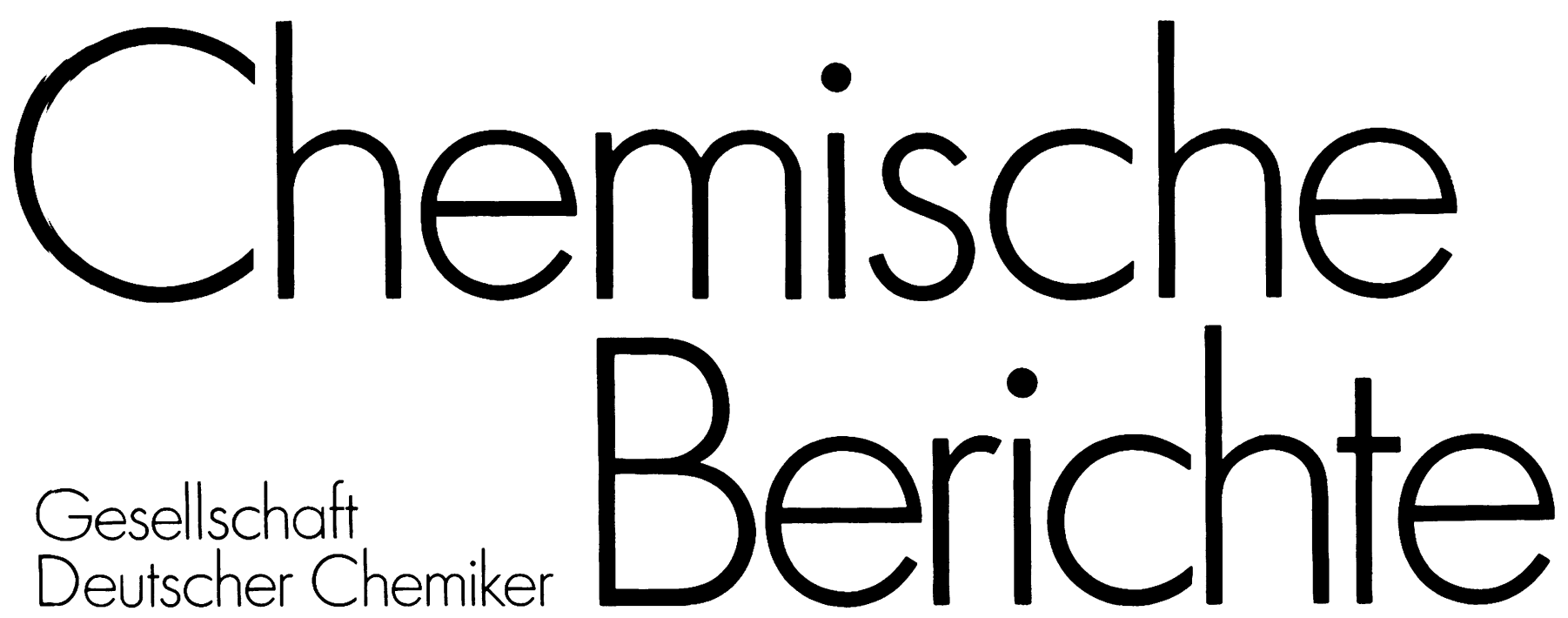

Founded 1868

August 1994 - Volume 127 - Issue 8 - Pages 1333-1554

Editorial Board: Wolfgang Beck

Rolf Gleiter

Klaus Hafner

Henning Hopf
Martin Jansen

Klaus Müllen

Heinrich Nöth

Werner Schroth
Helmut Schwarz

Werner Tochtermann

Heinrich Vahrenkamp

$\begin{array}{llll}\text { Managing Editor: } & \text { Robert Temme } & \text { Administration: } & \text { Hannelore Holm } \\ \text { Associate Editors: } & \text { Udo Eberhardt } & \text { Production: } & \text { Olga Olivecká }\end{array}$

Editorial Office:

Chemische Berichte

P.O. Box 101161

D-69451 Weinheim

Federal Republic of Germany

Telephone (internat.) $+496201606-255$

Telefax (internat.) +496201606-328
Publisher:

VCH Verlagsgesellschaft $\mathrm{mbH}$

P.O. Box 101161

D-69451 Weinheim

Federal Republic of Germany

Telephone (internat.) $+496201606-0$

Telefax (internat.) +496201606-328

Telex 465516 vchwh d
Die Chemischen Berichte erscheinen monatlich. Die Bezugsbedingungen für Mitglieder der Gesellschaft Deutscher Chemiker werden auf Anfrage von der Geschäftsstelle, Carl-Bosch-Haus, Varrentrappstraße 40-42, Postfach 900440, D-60444 Frankfurt, mitgeteilt. Bestellungen richten Sie bitte an Ihre Fachbuchhandlung oder unmittelbar an den Verlag. Abbestellungen nur bis spätestens 3 Monate vor Ablauf des Kalenderjahres. Die Lieferung erfolgt auf Rechnung und Gefahr des Empfängers. Gerichtsstand und Erfüllungsort: Weinheim.

Subscription rates (1994) including postage and handling:

Annual subscription: DM 1590.- (Germany), ÖS 11468.- (Austria), SFr 1468. - (Switzerland), GB£ 647. - (Great Britain), US\$ 1030.- (USA), DM 1617. - (elsewhere); single issue: DM 152.- (Germany; elsewhere on request). Back volumes of Chemische Berichte are available on microfilm (further information and prices on request).
For the USA and Canada: Chemische Berichte (ISSN 0009-2940) is published monthly by VCH Publishers, Inc., 303 N.W. 12th Avenue, Deerfield Beach FL 33442-1788; Telefax (305) 428-8201; Telephone (305) 428-5566 or (800) 422 8824. Second-class postage paid at Deerfield Beach FL 33441. Annual subscription price: US $\$ 1030.00$, single issue: US $\$ 107.00$ including postage and handling charges. Reduced rate for individual members of the American Chemical Society on request. - Printed in the Federal Republic of Germany.

U.S. POSTMASTER: Send address changes to Chemische Berichte, c/o VCH Publishers, Inc., 303 N.W. 12th Avenue, Deerfield Beach FL 33442-1788.

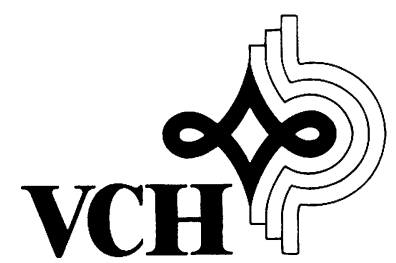


Adam, W., 1441

Alt, M., 1537

Bakken, P., 1469

Banert, K., 1479

Barnes, C. L., 1355

Beck, W., 1369

Behrens, U., 1399

Bestmann, H. J., 1501

Bilgin, B., 1543

Bläser, D., 1405

Boese, R., 1405

Bohnen, H., 1405

Bommers, S., 1359

Borowski, M., 1543

Dai, Y., 1533

de Meijere, A., 1511

Detert, H., 1529

Erker, G., 1551

Fischer, A., 1395

Flemming, S., 1489

Frey, R., 1387

Gabbert, G., 1363

Geismann, C., 1501

Gerold, A., 1547

Hafner, K., 1479

Hänel, R., 1459, 1469
Henrich, M., 1427

Herberich, G. E., 1401

Hopf, H., 1459, 1469

Hüffer, S., 1369

Hünig, S., 1495

Janiak, C., 1379

Jones, P. G., 1395

Kabbara, J., 1489

Kalinowski, H.-O., 1427

Kaschke, M., 1363

Katti, K. V., 1355

Keita, Y., 1495

Klintschar, G., 1411

Klug, P., 1441

Koert, U., 1447

Kolshorn, H., 1533

Krause, N., 1547

Krüger, C., 1551

Kühbauch, H., 1343

Kuhn, N., 1405

Kux, U., 1523

Lindner, E., 1343

Linti, G., 1387

Lipka, H., 1459

Maas, G., 1537
Mackert, P. J., 1479

Maier, G., 1427

Mayer, H. A., 1343

Mayer, W., 1529

Meier, H., 1529, 1533

Müller, C., 1395

Neda, I., 1395

Neh, H., 1489

Neidlein, R., 1523

Neumann, B., 1349

Nickisch, K., 1489

Nimmerfroh, N., 1479

Nolte, M., 1551

Peters, K., 1495

Pickardt, J., 1543

Pidun, U., 1447

Pinchuk, V. A., 1395

Polborn, K., 1369, 1387

Praefcke, K., 1543

Pritzkow, H., 1363

Psiorz, C., 1551

Rademacher, P., 1517

Reddy, V. S., 1355

Rose, B., 1529

Schleimer, R., 1437
Schmidbaur, H., 1359

Schmutzler, R., 1395

Schumann, I., 1349

Seipp, U., 1427

Shermolovich, Yu. G., 1395

Siebert, W., 1363

Sitzmann, H., 1335

Spaniol, T. P., 1401

Stammler, H.-G., 1349

Steffan, U., 1401

Sünkel, K., 1369

Trætteberg, M., 1459, 1469

Untiedt, S., 1511

Viebrock, H., 1399

von Schnering, H.-G., 1495

Wagner, H., 1447

Weber, L., 1349

Weiss, E., 1399

Westermann, J., 1489

Widhalm, M., 1411

Wieser, M., 1369

Wiesmann, R. F., 1517

Wolmershäuser, G., 1335

Würthwein, E.-U., 1437

Zimmermann, R., 1501

\section{Allgemeine Hinweise}

1. Die Chemischen Berichte (zu zitieren als Chem. Ber.) setzen seit 1946 die Berichte der Deutschen Chemischen Gesellschaft (zu zitieren als Ber. Disch. Chem. Ges.) fort. Die ..Berichte" enthalten Arbeiten aus dem Bereich der Anorganischen und Elementorganischen Chemic (Teil A) sowie der Organischen und Physikalisch-Organischen Chemie (Teil B). Bei der Organischen Chemie liegt der Schwerpunkt auf allgemeinen synthetischen Methoden und mechanistischen Problemen.

2. Die ,Berichte“ erscheinen monatlich; ein Register beschließt jeden Jahrgang.

3. Die Verantwortung für ihre Mitteilungen tragen die Verfasser selbst. - Der Korrespondenzautor ist durch einen hochgestellten Stern hervorgehoben. Die Zugehörigkeit der Autoren zu den Instituten ist durch hochgestellte Kleinbuchslaben markiert.

4. Es werden grundsätzlich nur Arbeiten aufgenommen, die vorher weder im Inland noch im Ausland veröffentlicht worden sind.

5. Eine Anweisung zur Abfassung von Manuskripten für die „Berichte“ wird auf Wunsch zugestellt (siehe auch Heft $1, \mathrm{~S}$. AI).

6. Manuskripte (in dreifacher Ausfertigung) sind zu senden an Redaktion der Chemischen Berichte, Dr. R. Temme. Postfach 101161. D-69451 Weinheim: Telefon (06201) 606-255.

7. Der Eingang der Abhandlungen wird den Autoren am Tage der Registrierung angezeigt.

8. Es werden nur Manuskripte in deutscher oder englischer Sprache aufgenommen. Allen Beiträgen ist eine knappe Zusammenfassung in Englisch voranzusiellen.

9. Der Autor muß das alleinige Urheberrechı besitzen. Mit der Annahme des Manuskriptes durch die Redaktion überträgt er der VCH Verlagsgesellschaft das ausschließliche Nutzungsrecht. insbesondere das Recht der Vervielfältigung wie Fotokopie. Mikrofilm - oder mit irgendeinem anderen Verfahren - oder das Manuskript in cine von Maschinen, insbesondere von Datenverarbeitungsmaschinen, verwendbare Sprache zu übergeben oder zu übersetzen (auch in fremde Sprachen).

10. Den Autoren werden 25 Sonderdrucke unentgeltlich portofrei zugesandt. Wünscht ein Autor mehr als 25 Abzüge, so ist dies auf dem Manuskript oder spätestens bei Rücksendung der Korrektur auf dieser zu vermerken. Den Autoren werden nur die Selbstkosten für die Zahl der die Freiexemplare überschreitenden Sonderabzüge berechnet.

11. Anfragen nach dem Verbleib nicht eingetroffener Berichte-Hefte oder Sonderdrucke sind zu richten an: $\mathrm{VCH}$ Verlagsgesellschaft mbH. Postfach 101161 , D-69451 Weinheim. Telefon (06201) 606-0.

\section{General Remarks}

1. Chemische Berichte (abbreviated Chem. Ber.; until 1946 Berichte der Deutschen Chemischen Gesellschaft, abbreviated Ber. Disch. Chem. Ges.) publishes articles on studies in inorganic and organometallic chemistry (Part A) as well as in organic and physical organic chemistry (Part B). In organic chemistry, emphasis is given to general synthetic methods and mechanistic problems.

2. Chemische Berichte is published monthly; the annual volume concludes with a List of Authors and a List of Contents.

3. Authors are solely responsible for their contributions. - The author to whom correspondence should be addressed should be indicated by an asterisk. Different addresses of coauthors should be given in lower-case letters.

4. The contents of a manuscript submitted to Chemische Berichte should not have been published previously, nor submitted to any other journal, except in the form of brief preliminary communication.

5. For the preparation of manuscripts, a copy of the "Instructions for Authors" is available on request (see also issue 1, p. AI).

6. Manuscripts should be submitted in triplicate to: Redaktion Chemische Berichte, Dr. R. Temme, P.O. Box 101161 , D-69451 Weinheim (FRG); Telephone (internat.) +49 $6201606-255$.

7. Acknowledgement of receipt of the manuscript will be confirmed on the day of registration.

8. Manuscripts may be submitted in German or in English. All contributions must be accompanied by a summary in English.

9. It is assumed that the authors concerned have the necessary authority for publication. Upon acceptance for publication. VCH Verlagsgesellschaft acquires all publishing rights including those of reprinting, translating or reproducing and distributing the manuscript in other forms (e.g. as photocopy. microfilm or in machine-readable form). Moreover, the provisions of the copyright law of the Federal Republic of Germany apply.

10. The author will receive one copy of the galley prools, together with the edited manuscript and a reprint order form; the author will receive 25 reprints free of charge and may order additional copies at cost price.

11. All inquiries concerning journals or reprints should be addressed to: $\mathrm{VCH}$ Verlagsgesellschaft mbH. P.O. Box 101161, D-69451 Weinheim (FRG) Telephone (internat.) $+496201606-0$. 
Sitzmann*, H., Wolmershäuser, G.

Wismutverbindungen mit voluminösen, mehrfach alkylierten Cyclopentadienyl-Liganden

Bismuth Compounds with Crowded Multiply Alkylated Cyclopentadienyl Ligands

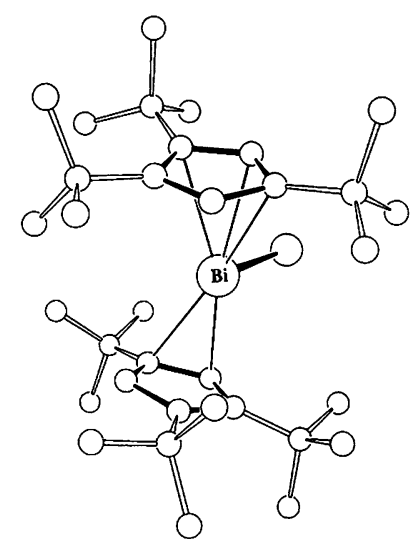

1343 Lindner*, E., Kühbauch, H., Mayer, H. A.

Darstellung, Eigenschaften und Reaktionen Metallenthaltender Heterocyclen, XC. - Reaktivität unterschiedlich aktivierter Alkine gegenüber Ruthenium und Osmium-Komplexen des Typs $\left(\eta^{2}-\mathrm{C}_{2} \mathrm{H}_{4}\right) \mathrm{M}(\mathrm{CO})_{4}$ Preparation, Properties, and Reactions of Metal-Containing Heterocycles, XC. - Reactivity of Differently Activated Alkynes toward Ruthenium and Osmium Complexes of the Type $\left(\eta^{2}-\mathrm{C}_{2} \mathrm{H}_{4}\right) \mathrm{M}(\mathrm{CO})_{4}$

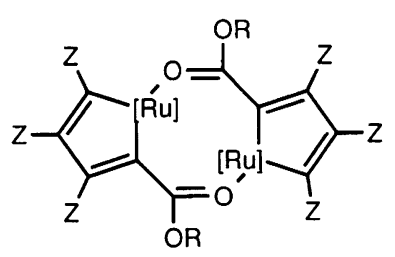

$3 a, b$

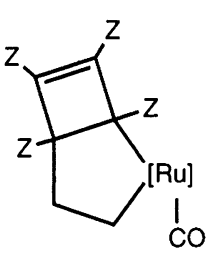

4 c-e<smiles>[Z]C1=C([Z])[In](C)C1</smiles>

$5 \mathrm{f}, \mathrm{g}$
Weber*, L., Schumann, I., Stammler, H.-G., Neumann, B.

Übergangsmetallsubstituierte Acylphosphane und Phosphaalkene, XXI. - Isophosphaalkine als $\mu_{3}$-Liganden in Übergangsmetallkomplexen

Transition-Metal-Substituted Acylphosphanes and Phosphaalkenes, XXI. - Isophosphaalkynes as $\mu_{3}$ - Ligands in Transition Metal Complexes
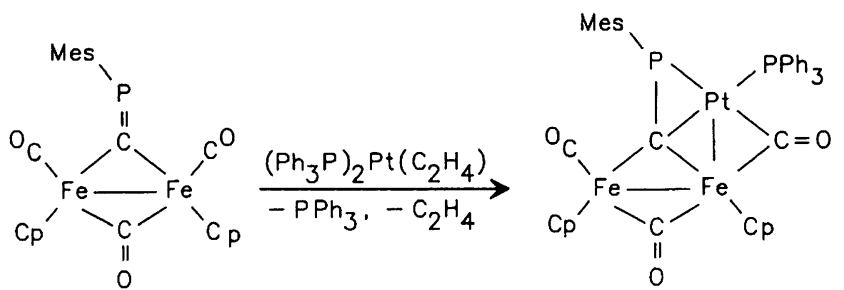

1355 Reddy, V. S., Katti*, K. V., Barnes, C. L.

Übergangsmetall-Chemie von Hauptgruppen-Element-Hydraziden, 9. - Platin-Komplexe von Diphosphanylhydraziden $\mathrm{R}_{2} \mathrm{PN}(\mathrm{Me}) \mathrm{N}(\mathrm{Me}) \mathrm{PR}_{2} \cdot \mathrm{PtCl}_{2}(\mathrm{R}=$ $\mathrm{OPh}, \mathrm{o}-\mathrm{OC}_{6} \mathrm{H}_{4} \mathrm{CH}_{2} \mathrm{CHCH}_{2}$ )

Transition Metal Chemistry of Main Group Hydrazides, 9. - Platinum Complexes of Diphosphanylhydrazides $\mathrm{R}_{2} \mathrm{PN}(\mathrm{Me}) \mathrm{N}(\mathrm{Me}) \mathrm{PR}_{2} \cdot \mathrm{PtCl}_{2}(\mathrm{R}=\mathrm{OPh}$, $o-\mathrm{OC}_{6} \mathrm{H}_{4} \mathrm{CH}_{2} \mathrm{CHCH}_{2}$ )<smiles>CN1[C@@H](Cl)[C@@H](Cl)P([Pb])N1C</smiles>

$\mathbf{R}=\mathbf{P h}$

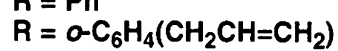

\section{9 \\ Bommers, S., Schmidbaur*, H.}

Ein Syntheseweg zu 1,3-Bis(trisilylmethyl)disiloxane $\left[\left(\mathrm{H}_{3} \mathrm{Si}_{3}\right)_{3} \mathrm{CSiH}_{2}\right]_{2} \mathrm{O}$ - dem Octasila-Analogen von Dineopentylether $\left[\left(\mathrm{H}_{3} \mathrm{C}\right)_{3} \mathrm{CCH}_{2}\right]_{2} \mathrm{O}$

A Synthetic Pathway to 1,3-Bis(trisilylmethyl)disiloxane $\left[\left(\mathrm{H}_{3} \mathrm{Si}\right)_{3} \mathrm{CSiH}_{2}\right]_{2} \mathrm{O}$ - the Octasila Analogue of Dineopentyl Ether $\left[\left(\mathrm{H}_{3} \mathrm{C}\right)_{3} \mathrm{CCH}_{2}\right]_{2} \mathrm{O}$

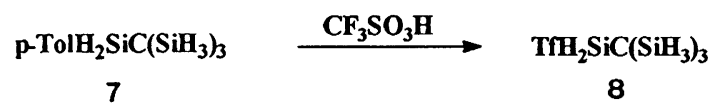

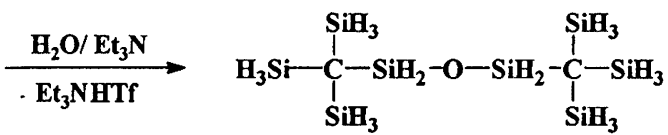


1363 Gabbert, G., Pritzkow, H., Kaschke, M., Siebert*, W.

Ein neuer Zugang zu 1,2- und 1,3-Diborol

A New Approach to 1,2- and 1,3-Diborole

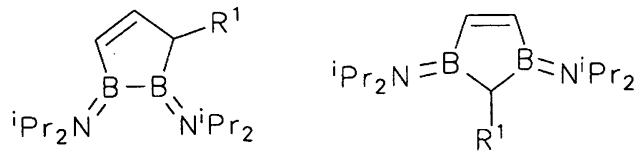

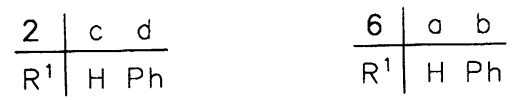

Hüffer, S., Wieser, M., Polborn, K., Sünkel, K., Beck* ${ }^{*}$.

Kohlenwasserstoffverbrückte Komplexe, XXX. Nucleophile Addition von Carbonylmetallaten an kationische Vinyl-, Dien-, Dienyl- und Trien-Komplexe von Eisen, Ruthenium und Cobalt: Zwei-, drei-, vierund fünfkernige Komplexe mit $\sigma, \sigma$ - und $\sigma, \pi$-Kohlenwasserstoffbrücken

Hydrocarbon-Bridged Complexes, XXX. - Nucleophile Addition of Carbonylmetallates to Cationic Vinyl, Diene, Dienyl and Triene Complexes of Iron, Ruthenium and Cobalt: Di-, Tri-, Tetra- and Pentametallic Complexes with $\sigma, \sigma$ - and $\sigma, \pi$-Hydrocarbon Bridges
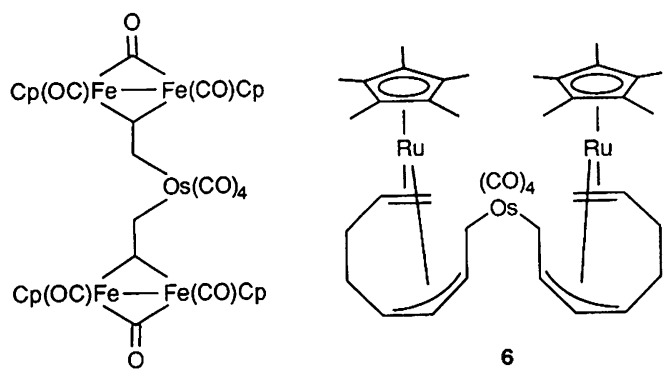

3
Janiak, C.

Binäre [Hydrotris(1,2,4-triazolyl)borat]metall-Komplexe $\left[\mathrm{M}\left\{\mathrm{HB}\left(\mathrm{C}_{2} \mathrm{H}_{2} \mathrm{~N}_{3}\right)_{3}\right\}_{2}\right]$ mit $\mathrm{M}=\mathrm{Fe}, \mathrm{Co}, \mathrm{Cu}, \mathrm{Zn}$ : Synthese, Charakterisierung, Magnetochemie und Röntgenstruktur von $\left[\mathrm{Cu}\left\{\mathrm{HB}\left(\mathrm{C}_{2} \mathrm{H}_{2} \mathrm{~N}_{3}\right)_{3}\right\}_{2}\right] \cdot 4$ $\mathrm{CH}_{3} \mathrm{OH}$ und $\mathrm{K}\left[\mathrm{HB}\left(\mathrm{C}_{2} \mathrm{H}_{2} \mathrm{~N}_{3}\right)_{3}\right] \cdot 2 \mathrm{H}_{2} \mathrm{O}$

Binary [Hydrotris(1,2,4-triazolyl)borate]metal Complexes $\left[\mathrm{M}\left\{\mathrm{HB}\left(\mathrm{C}_{2} \mathrm{H}_{2} \mathrm{~N}_{3}\right)_{3}\right\}_{2}\right]$ with $\mathrm{M}=\mathrm{Fe}, \mathrm{Co}, \mathrm{Cu}, \mathrm{Zn}$ : Synthesis, Charakterization, Magnetochemistry, and X-ray Structure of $\left[\mathrm{Cu}\left\{\mathrm{HB}\left(\mathrm{C}_{2} \mathrm{H}_{2} \mathrm{~N}_{3}\right)_{3}\right\}_{2}\right] \cdot 4 \mathrm{CH}_{3} \mathrm{OH}$ and $\mathrm{K}\left[\mathrm{HB}\left(\mathrm{C}_{2} \mathrm{H}_{2} \mathrm{~N}_{3}\right)_{3}\right] \cdot 2\left(\mathrm{H}_{2} \mathrm{O}\right)$

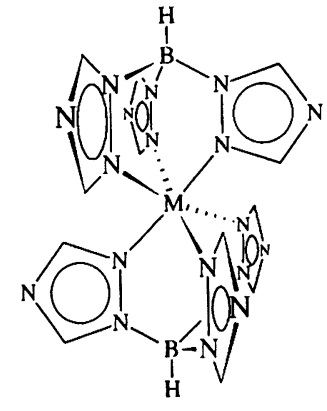

\section{Linti*, G., Frey, R., Polborn, K.}

Zur Chemie des Galliums, IV. - Darstellung und Strukturen monomerer (2,2,6,6-Tetramethylpiperidino)gallane

On the Chemistry of Gallium Compounds, IV. - Synthesis and Structure of Monomeric (2,2,6,6-Tetramethylpiperidino)gallanes
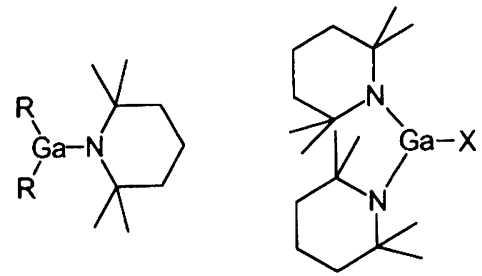

$R=M e, E t$<smiles>[R][R]Nc1ccccc1</smiles>

Pinchuk, V. A., Neda, I., Müller, C., Fischer, A., Jones, P. G., Shermolovich, Yu. G., Schmutzler*, R.

2-Chlor-2-phenyl-2-( $p$-tolylthio)-1,3-diorgano-1,3,2 $2 \lambda^{5}$ diazaphosphetidin-4-one

2-Chloro-2-phenyl-2-( $p$-tolylthio)-1,3-diorgano-1,3,2 $\lambda^{5}$ diazaphosphetidin-4-ones 
Über Metallalkyl- und -aryl-Verbindungen, 53. - Synthese und Struktur von [9,10-Dihydroanthracenylnatrium(tmeda) $]_{n}$ und [9,10-Dihydroanthracenylnatrium$($ pmdta) $]($ tmeda $=$ Tetramethylethylendiamin, pmdta $=$ Pentamethyldiethylentriamin)

On Alkyl- and Arylmetal Compounds, 53. - Synthesis and Structure of [9,10-Dihydroanthracenylsodium(tmeda) $]_{n}$ and [9,10-Dihydroanthracenylsodium(pmdta)] $($ tmeda $=$ tetramethylethylenediamine, pmdta $=$ pentamethyldiethylenetriamine)

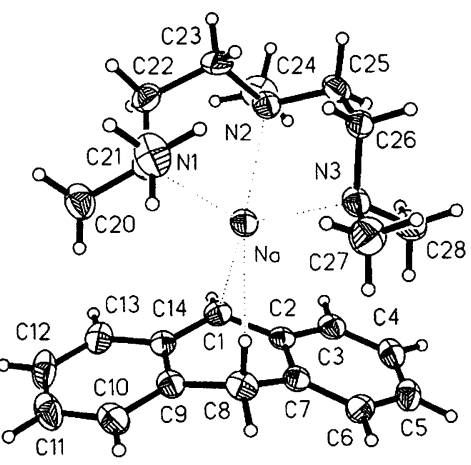

1401 Herberich*, G. E., Spaniol, T. P., Steffan, U.

3-Borolene mit diastereotopen Seiten: Synthese von 4(Dialkylamino)-4-boratricyclo[5.2.1.02,6]dec-2(6)-enen und Struktur des Dimethylamino-Derivats

3-Borolenes with Diastereotopic Faces: Synthesis of 4(Dialkylamino)-4-boratricyclo[5.2.1. $0^{2,6}$ dec-2(6)-enes and Structure of the Dimethylamino Derivative

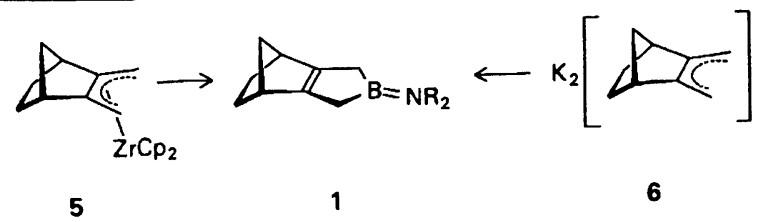

a: $R=M e, b: R=E t, c: R=P r$

1405 Kuhn*, N., Bohnen, H., Bläser, D., Boese, R., Derivate des Imidazols, XI. - $\left(\mathrm{C}_{8} \mathrm{H}_{14} \mathrm{~N}_{2}\right) \mathrm{M}(\mathrm{CO})_{5}$ $(\mathrm{M}=\mathrm{Mo}, \mathrm{W})-$ Terminale Koordination eines Olefins in Pentacarbonylmetall-Komplexen

Imidazole Derivatives, $\mathrm{XI} .-\left(\mathrm{C}_{8} \mathrm{H}_{14} \mathrm{~N}_{2}\right) \mathrm{M}(\mathrm{CO})_{5}(\mathrm{M}=$ Mo, W) - Terminal Coordination of an Olefin in Pentacarbonyl Metal Complexes<smiles>C=C1N(C)C(C)=C(C)N1C</smiles><smiles>CC(=O)OCCC(=O)OC(C)(C)C</smiles>

5

$M=M o(a), W(b)$

6

Teil B: Organische Chemie / Part B: Organic Chemistry

1411

Widhalm*, M., Klintschar, G.

Synthese und Stereochemie chiraler Makrocyclen, die 1,2-Bis(phenylphosphanyl)benzol-Einheiten enthalten Synthesis and Stereochemistry of Chiral Macrocycles Including a 1,2-Bis(phenylphosphanyl)benzene Unit

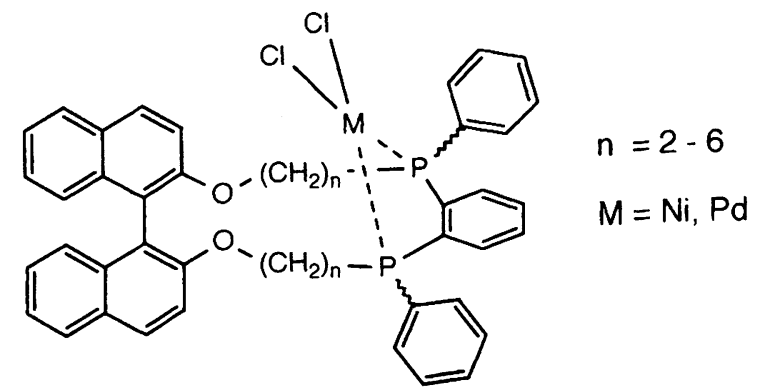

1427 Maier*, G., Seipp, U., Kalinowski, H.-O.,

\section{Henrich, $M$.}

Festkörper- ${ }^{13} \mathrm{C}-\mathrm{NMR}$-Spektroskopie, 1. - Titantetrachlorid-Komplexe von Diketonen: Ihre Bedeutung für Hydrid-Reduktionen und ihre Festkörper-NMRSpektren

Solid-State ${ }^{13}$ C-NMR Spectroscopy, 1. - Titanium Tetrachloride Complexes of Diketones: Their Importance for Hydride Reductions and Their Solid-State NMR Spectra

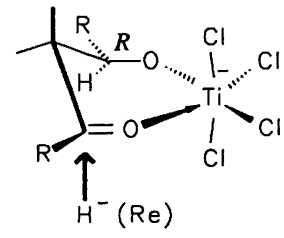

32 
2,6-Disubstituierte 4-Aminopyridine aus 1,3-Dialkoxy2-azapropenylium-Salzen und $N$-Methyl-4-piperidonEnaminen

2,6-Disubstituted 4-Aminopyridines from 1,3-Dialkoxy-2-azapropenylium Salts and $N$-Methyl-4-piperidone Enamines

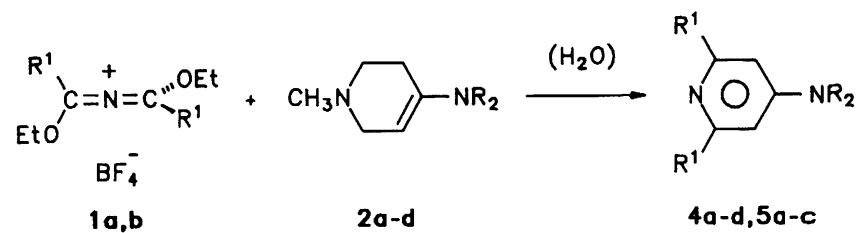

\section{Adam*, W., Klug, P.}

Regio- und Diastereoselektive Synthese von Stannylepoxyalkoholen durch direkte Hydroxy-Epoxidierung von Vinylstannanen

Regio- and Diastereoselective Synthesis of Stannyl Epoxy Alcohols by Direct Hydroxy Epoxidation of Vinylstannanes

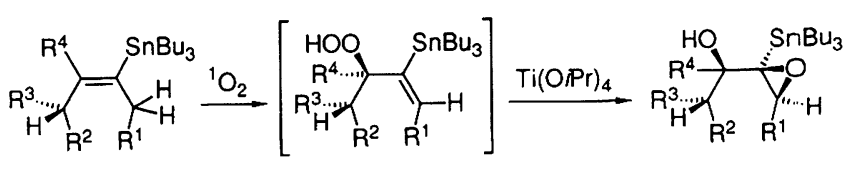

$\mathrm{R}^{1}=\mathrm{R}^{2}=\mathrm{H},\left[\mathrm{CH}_{2}\right]_{n}(n=1,2) ; \mathrm{R}^{3}=\mathrm{H}, \mathrm{OH} ; \mathrm{R}^{4}=\mathrm{H}, \mathrm{Me}$
Koert*, U., Wagner, H., Pidun, U.

Stereoselektive Additionen chiraler funktionalisierter Organozink-Reagenzien an achirale und chirale Aldehyde: Ein „Matched-Mismatched“-Fall in der Organozink-Chemie

Stereoselective Additions of Chiral, Functionalized Organozinc Reagents to Achiral and Chiral Aldehydes: a Matched-Mismatched Case in Organozinc Chemistry

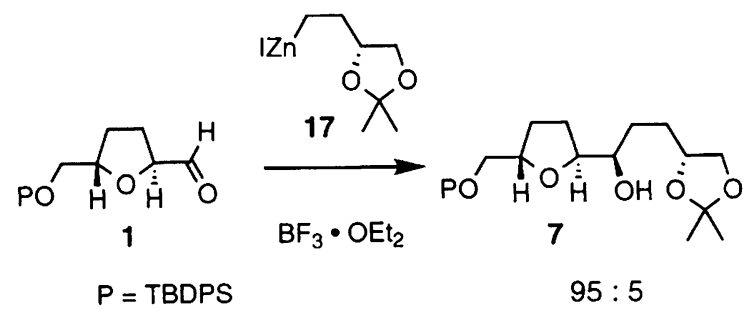

$95: 5$

1459 Trætteberg, M., Hopf*, H., Lipka, H., Hänel, R. Hochsubstituierte 1,3-Diene, IV. - Eine experimentelle und theoretische Studie über die stereochemischen Eigenschaften von 2-tert-Butyl-1,3-butadien und 2,3-Ditert-butyl-1,3-butadien

Highly Substituted 1,3-Dienes, IV. - An Experimental and Theoretical Study of the Stereochemical Properties of 2-tert-Butyl-1,3-butadiene and 2,3-Di-tert-butyl-1,3-butadiene

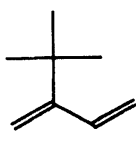

1

dihedral angle $(\varphi): \quad 32.1^{\circ}$

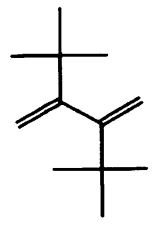

2

$101.5^{\circ}$
Trætteberg, M., Bakken, P., Hopf*, H., Hänel, R.

Hochsubstituierte 1,3-Diene, V. - Eine theoretische Studie über die stereochemischen Effekte von tert-Butyl-Substituenten an 1,3-Butadien

Highly Substituted 1,3-Dienes, V. - A Theoretical Study of the Stereochemical Effects of tert-Butyl Substituents in 1,3-Butadiene
Mackert, P. J., Hafner*, K., Nimmerfroh, N., Banert, $K$.

Synthese, Struktur und Reaktivität von Cyclopentaanellierten 1,2,3,4-Tetrazinen

Synthesis. Structure and Reactivity of Cyclopenta-annulated 1,2,3,4-Tetrazines

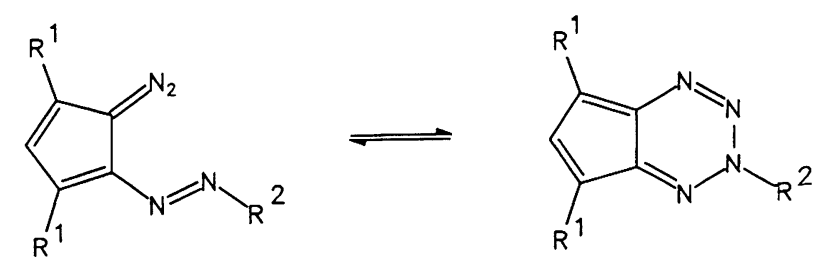

2

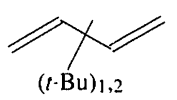

3

$R^{1}: H_{0}\left(\mathrm{CH}_{3}\right)_{3} \mathrm{C}: \mathrm{R}^{2}: \mathrm{Ar}$ 
Kabbara*, J., Flemming, S., Nickisch, K.,

Neh, H., Westermann, J.

Kupfer-katalysierte konjugierte Addition von Trimethylaluminium an $\alpha, \beta$-ungesättigte Ketone

Copper-Catalyzed Conjugate Addition of Trimethylaluminium to $\alpha, \beta$-Unsaturated Ketones

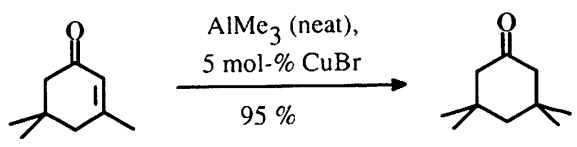

1495 Hünig*, S., Keita, Y., Peters, K., von Schnering, H.-G.

Stereoselektive Protonierung von Carbanionen, 3. 1,3-Dioxolan-4-one und 1,3-Oxazolidin-4-one: Synthesen und diastereoselektive Protonierung ihrer Anionen Stereoselective Protonation of Carbanions, 3. - 1,3Dioxolan-4-ones and 1,3-Oxazolidine-4-ones: Syntheses and Diastereoselective Protonation of their Anions

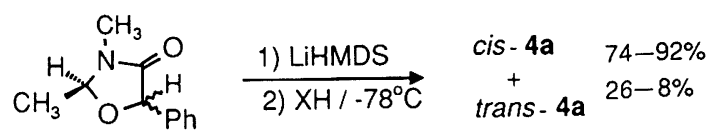

cis/trans-4a

\section{Bestmann*, H. J., Geismann, C.,} Zimmermann, R.

Kumulierte Ylide, 22. - Umsetzungen von (Triphenylphosphoranyliden)ethenon mit Halogenverbindungen und ihre präparative Anwendung

Cumulated Ylides, 22. - Reactions of (Triphenylphosphoranylidene)ethenone with Halogen Compounds and their Preparative Application

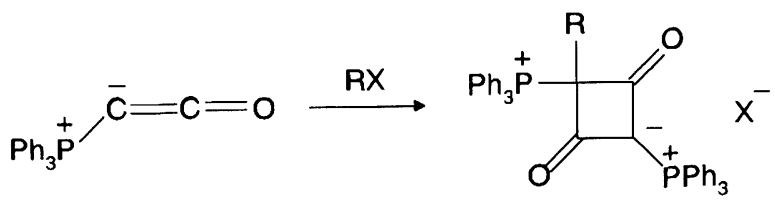

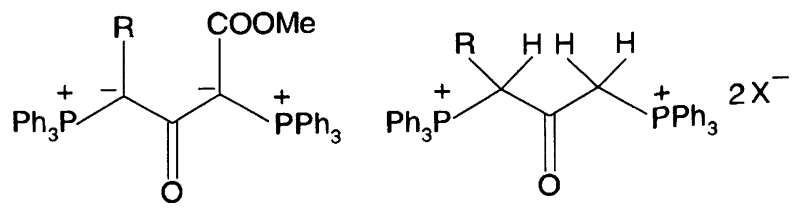

1511 Untiedt, S., de Meijere*, A.

Neue Cyclopropyl-Bausteine für die organische Synthese, 28. - Pd(0)-katalysierte Kupplung von Cyclopropenylzink-chloriden und Cyclopropenylstannanen. - Eine neue effiziente Synthese von 1-Phenyl-, 1-Ethenyl- und 1-Ethinyl-1-cyclopropenen

New Cyclopropyl Building Blocks for Organic Synthesis, 28. - Pd(0)-Catalysed Coupling of Cyclopropenylzinc Chlorides and Cyclopropenylstannanes. - A New Efficient Synthesis of 1-Phenyl-, 1-Ethenyl-, and 1Ethynyl-1-cyclopropenes

1517 Wiesmann, R. F., Rademacher*, P.

Transanulare Wechselwirkungen in difunktionellen mittleren Ringen, 6. - Spektroskopische Untersuchungen von bicyclischen Aminoketonen

Transanular Interactions in Difunctional Medium Rings, 6. - Spectroscopic Investigations of Bicyclic Amino Ketones
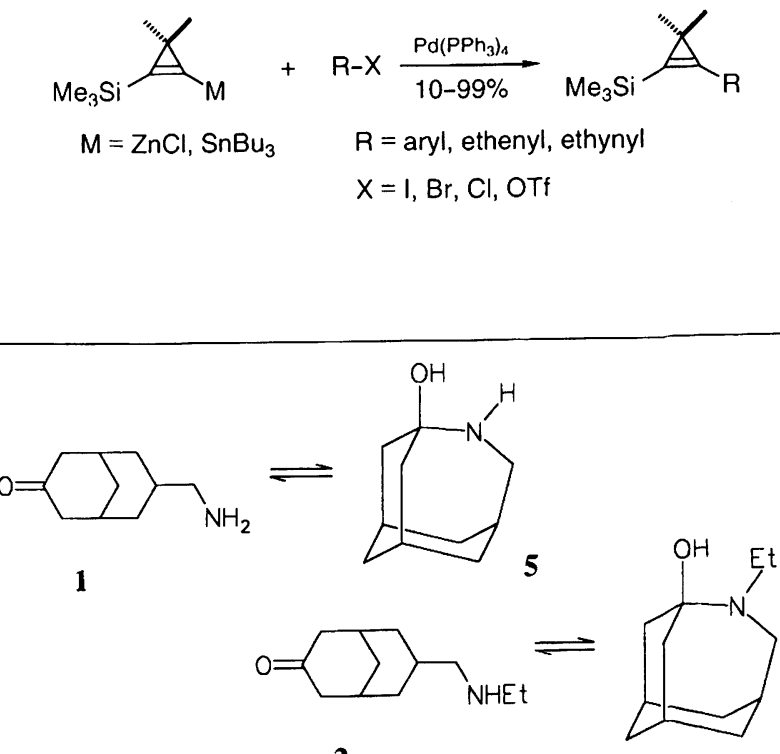

2

Neidlein*, R., Kux, U.

Neue 1,6-Methano[10]annulen-Derivate mit ,parallelen Dreifachbindungen"

New 1,6-Methano[10]annulene Derivatives with "Parallel Triplebonds"
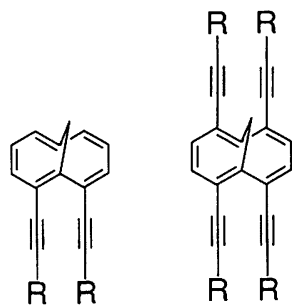

$\mathrm{R}=\mathrm{C}_{6} \mathrm{H}_{5^{-}} ; \mathrm{Me}_{3} \mathrm{Si}-$ 
Herstellung von 1,5-Cyclooctadiin und 1,3,5,7-Cyclooctatetraen aus 1,5-Cyclooctadien

Preparation of 1,5-Cyclooctadiyne and 1,3,5,7-Cyc-

looctatetraene from 1,5-Cyclooctadiene

1533 Dai, Y., Kolshorn, H., Meier*, H.

Dithiametacyclophane mit Dreifachbindungen

Dithiametacyclophanes with Triple Bonds
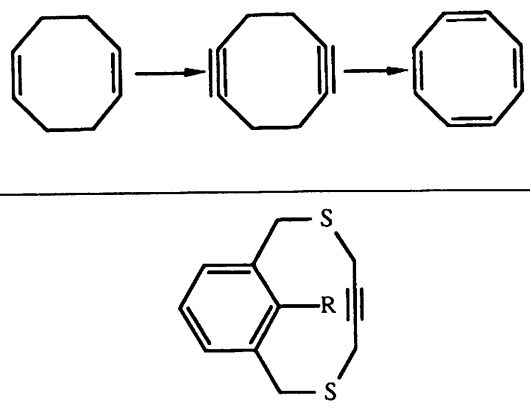

Alt, M., Maas*, G.

Übergangsmetall-katalysierte Reaktionen von ungesättigten $\alpha$-Diazo- $\alpha$-(trimethylsilyl)essigestern mit Carbonylverbindungen

Transition Metal Catalyzed Reactions of Unsaturated $\alpha$-Diazo- $\alpha$-(trimethylsilyl)acetates with Carbonyl Compounds
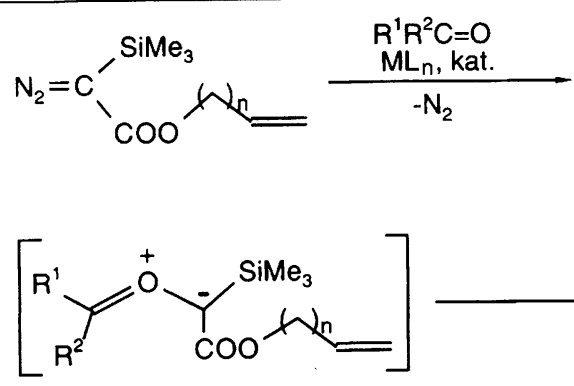

14

\section{Notizen / Notes}

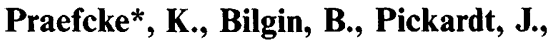
Borowski, M.

Flüssig-kristalline Verbindungen, 86. - Das erste scheibenförmige dinucleare Platin-Mesogen

Liquid-crystalline Compounds, 86. - The First Disc-

Shaped Dinuclear Platinum Mesogen
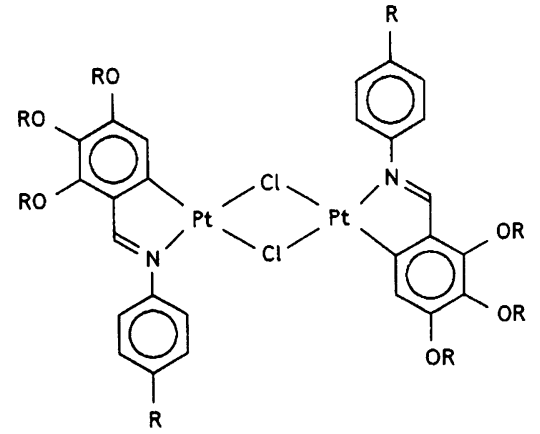

$R=\mathrm{C}_{6} \mathrm{H}_{13} \quad \mathrm{Cr} 91.7^{\circ} \mathrm{C}\left\{\mathrm{N}_{\mathrm{D}} 54.4^{\circ} \mathrm{C}\right\}$ Iso

\section{Kurzmitteilung / Short Communication}

\section{1 \\ Erker*, G., Psiorz, C., Krüger, C., Nolte, M.}

Synthese eines neuartigen anellierten $\mathrm{C}_{1}$-überbrückten ansa-Metallocen-Systems

Synthesis of a Novel Annulated $\mathrm{C}_{1}$-Bridged ansa-Metallocene System

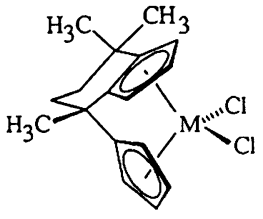

$6(\mathrm{a}: \mathrm{M}=\mathrm{Ti} ; \mathrm{b}: \mathrm{M}=\mathrm{Zr})$ 


\title{
Nucleophile Addition von Carbonylmetallaten an kationische Vinyl-, Dien-, Dienyl- und Trien-Komplexe von Eisen, Ruthenium und Cobalt: Zwei-, drei-, vier- und fünfkernige Komplexe mit $\sigma, \sigma-$ und $\sigma, \pi$-Kohlenwasserstoffbrücken ${ }^{2 / 3}$
}

\author{
Stephan Hüffer, Michael Wieser, Kurt Polborn ${ }^{[2]}$, Karlheinz Sünkel ${ }^{[2]}$ und Wolfgang Beck* \\ Institut für Anorganische Chemie der Universität München, \\ Meiserstraße 1, 80333 München, Germany
}

Eingegangen am 11. Februar 1994

Key Words: Tungsten complexes / Rhenium complexes / Iron complexes / Ruthenium complexes / Osmium complexes / Cobalt complexes / Vinyl / Cyclohexadienyl / Cycloheptadienyl / Cyclooctatrienyl /

Hydrocarbon-bridged complexes

Hydrocarbon-Bridged Complexes, $\mathrm{XXX}^{[1]}$. - Nucleophilic Addition of Carbonylmetallates to Cationic Vinyl, Diene, Dienyl and Triene Complexes of Iron, Ruthenium and Cobalt: Di-, Tri-, Tetra- and Pentametallic Complexes with $\sigma, \sigma-$ and $\sigma, \pi-$ Hydrocarbon Bridges ${ }^{\text {tr }}$

The reactions of $\left[\operatorname{Re}(\mathrm{CO})_{5}\right]^{-},\left[\mathrm{Ru}(\mathrm{CO})_{2} \mathrm{Cp}\right]^{-}$, and $\left[\mathrm{Os}(\mathrm{CO})_{4}\right]^{2-}$ with $\left[\mathrm{Cp}_{2}(\mathrm{OC})_{2} \mathrm{Fe}_{2}(\mu-\mathrm{CO})\left(\mu-\eta^{1}: \eta^{2}-\mathrm{CH}=\mathrm{CH}_{2}\right)\right]^{+},\left[\mathrm{Cp} * \mathrm{Ru}\left(\eta^{2}: \eta^{4}-\right.\right.$ $1,3,7$-octatriene $)]^{+}, \quad\left[(\mathrm{OC}) \mathrm{Fe}\left(\eta^{4} \text {-diene }\right)\left(\eta^{5} \text {-cycloheptadienyl }\right)\right]^{+}$, and $\left[\mathrm{CpCo}\left(\eta^{5} \text {-cyclodienyl }\right)\right]^{+}$give the nucleophilic adducts whereas with $\left[\mathrm{Mn}(\mathrm{CO})_{5}\right]^{-},\left[\mathrm{W}(\mathrm{CO})_{3} \mathrm{Cp}\right]^{-}$, and $\left[\mathrm{Fe}(\mathrm{CO})_{2} \mathrm{Cp}\right]^{-}$ formation of the corresponding $\mathrm{C}-\mathrm{C}$ coupling products and of the metal-metal-bonded dimers is observed. The structures of $\mathrm{Cp}^{*} \mathrm{Ru}\left(\mu-\eta^{1}: \eta^{2}: \eta^{3}-1,5\right.$-octadienyl) $\operatorname{Re}(\mathrm{CO})_{5} \quad(4),\left[\mathrm{Cp}{ }^{*}\right.$ $\operatorname{Ru}\left(\mu-\eta^{1}: \eta^{2}: \eta^{3}-1,5\right.$-octadienyl $\left.)\right]_{2} \mathrm{Os}(\mathrm{CO})_{4}(6)$, and of (OC)$\mathrm{Fe}\left(\eta^{4}-1,3\right.$-cyclohexadiene $)\left(\mu-\eta^{1}: \eta^{4}-1,3\right.$-cycloheptadiene) $\operatorname{Re}(\mathrm{CO})_{5}(9)$ have been determined by X-ray diffraction.
In unseren vorangegangenen Arbeiten ${ }^{[1,2]}$ konnten wir zeigen, daß die nucleophile Addition von Carbonylmetallaten an kationische Übergangsmetall-Komplexe mit ungesättigten Kohlenwasserstoff-Liganden eine effektive Methode zum gezielten Aufbau von di- und trimetallischen Kohlenwasserstoff-verbrückten Komplexen darstellt. Im allgemeinen werden die dabei auftretenden Regioselektivitäten durch die semiempirischen Regeln von Davies, Green und Mingos ${ }^{[3]}$ beschrieben. Im folgenden wird gezeigt, daß sich mit $\left[\mathrm{Cp}_{2}(\mathrm{OC})_{2} \mathrm{Fe}_{2}(\mu-\mathrm{CO})\left(\mu-\eta^{1}: \eta^{2}-\mathrm{CH}=\mathrm{CH}_{2}\right)\right]^{+}$in gezielter Weise drei-, vier- und fünfkernige Komplexe aufbauen lassen. Eine ungewöhnliche Regioselektivität wird bei der Addition von Carbonylmetallaten an $\left[(\mathrm{OC}) \mathrm{Fe}\left(\eta^{4}-\right.\right.$ Dien $)\left(\eta^{5}\right.$-Cycloheptadienyl $\left.)\right]^{+}$beobachtet. Die bisher von uns eingesetzten Kationen ${ }^{[1.2]}$ enthielten immer elektronenziehende und somit die Elektrophilie verstärkende Carbonyl-Liganden. Es erschien deshalb von Interesse, die Kationen $\left[\mathrm{Cp} \mathrm{p}^{*} \mathrm{Ru}\left(\eta^{2}: \eta^{4}-1,3,7-\text { Octatrien }\right)\right]^{+}$und $\left[\mathrm{CpCo}\left(\eta^{5}-\mathrm{Cyclo-}\right.\right.$ dienyl) $]^{+}$, welche keine Carbonyl-Liganden enthalten, einzusetzen. Auch die mit der nucleophilen Addition konkurrierende Elektronenübertragung vom Anion zum Kation kann unter C-C-Kupplung der koordinierten Liganden zu Kohlenwasserstoff-verbrückten Komplexen füh$\operatorname{ren}^{[2,4]}$.

\section{Ergebnisse und Diskussion}

Nucleophile wie Hydrid, Lithiumorganyle, Phosphane, Methanolat, Diethylmalonat und Methyl(phenylsulfonyl)- sulfonat addieren sich an das $\beta$-Kohlenstoffatom des VinylLiganden von $\left[\mathrm{Cp}_{2} \mathrm{Fe}_{2}(\mathrm{CO})_{2}(\mu-\mathrm{CO})\left(\mu-\eta^{\prime}: \eta^{2}-\mathrm{CH}=\mathrm{CH}_{2}\right)\right]^{+[5,6]}$. In der gleichen Weise reagiert $\left[\mathrm{Cp}_{2} \mathrm{Fe}_{2}(\mathrm{CO})_{2}(\mu-\mathrm{CO})(\mu-\right.$ $\left.\left.\eta^{1}: \eta^{2}-\mathrm{CH}=\mathrm{CH}_{2}\right)\right]^{+}$mit den Carbonylmetallaten $\left[\mathrm{Re}(\mathrm{CO})_{5}\right]^{-}$ und $\left[\mathrm{CpRu}(\mathrm{CO})_{2}\right]^{-} \mathrm{zu}$ den dreikernigen Komplexen 1 und 2. Die Folgeprodukte der Elektronenübertragung zwischen Anion und Kation, $\operatorname{Re}_{2}(\mathrm{CO})_{10}$ bzw. $\left[\mathrm{Cp} \mathrm{Ru}(\mathrm{CO})_{2}\right]_{2}$, entstehen dabei nur in geringen Mengen. Analog wird $\left[\mathrm{Cp}_{2} \mathrm{Fe}_{2}-\right.$ $\left.(\mathrm{CO})_{2}(\mu-\mathrm{CO})\left(\mu-\eta^{1}: \eta^{2}-\mathrm{CH}=\mathrm{CH}_{2}\right)\right]^{+}$zweimal an $\left[\mathrm{Os}(\mathrm{CO})_{4}\right]^{2-}$ unter Bildung des fünfkernigen Komplexes 3 addiert.

Setzt man $\left[\mathrm{Cp}_{2} \mathrm{Fe}_{2}(\mathrm{CO})_{2}(\mu-\mathrm{CO})\left(\mu-\eta^{1}: \eta^{2}-\mathrm{CH}=\mathrm{CH}_{2}\right)\right]^{+}$ dagegen mit den Carbonylmetallaten $\left.\left[\mathrm{CpW}(\mathrm{CO})_{3}\right)\right]^{-}$, $\left.\left[\mathrm{Mn}(\mathrm{CO})_{5}\right)\right]^{-}$oder $\left.\left[\mathrm{CpFe}(\mathrm{CO})_{2}\right)\right]^{-}$um, so bildet sich schon bei Temperaturen um ca. $-50^{\circ} \mathrm{C}$ ein ziegelroter Niederschlag, der in allen gängigen, polaren und unpolaren Lösungsmitteln schwerlöslich ist. Gleichzeitig entstehen die Folgeprodukte des Elektronentransfers zwischen Kation und Anion $\left.\left[\mathrm{CpW}(\mathrm{CO})_{3}\right)\right]_{2}, \mathrm{Mn}_{2}(\mathrm{CO})_{10}$ bzw. $\left[\mathrm{CpFe}(\mathrm{CO})_{2}\right]_{2}{ }^{[4]}$. Auch bei der Reduktion von $\left[\mathrm{Cp}_{2} \mathrm{Fe}_{2}-\right.$ $\left.(\mathrm{CO})_{2}(\mu-\mathrm{CO})\left(\mu-\eta^{1}: \eta^{2}-\mathrm{CH}=\mathrm{CH}_{2}\right)\right]^{+}$mit Natriumamalgam entsteht die ziegelrote Verbindung, die vermutlich das C-C-Kupplungsprodukt $\quad\left[\mathrm{Cp}_{2} \mathrm{Fe}_{2}(\mathrm{CO})_{2}(\mu-\mathrm{CO})(\mu-\right.$ $\left.\left.\eta^{1}: \eta^{1}-\mathrm{CH}-\mathrm{CH}_{2}-\right)\right]_{2}$ ist. Erwähnt seien hier die von Casey, Marder et al. beschriebenen kationischen Tetraeisen-Komplexe mit konjugierten $\mathrm{C}_{3^{-}}{ }^{[7]}, \mathrm{C}_{5^{-}}$und $\mathrm{C}_{7}$-Brücken ${ }^{[8,9]}$. Als metallorganische Cyanine mit hohen Extinktionskoeffizienten beanspruchen diese Verbindungen großes Interesse. Außerdem sind von diesen Komplexen Elektronentransfer- 


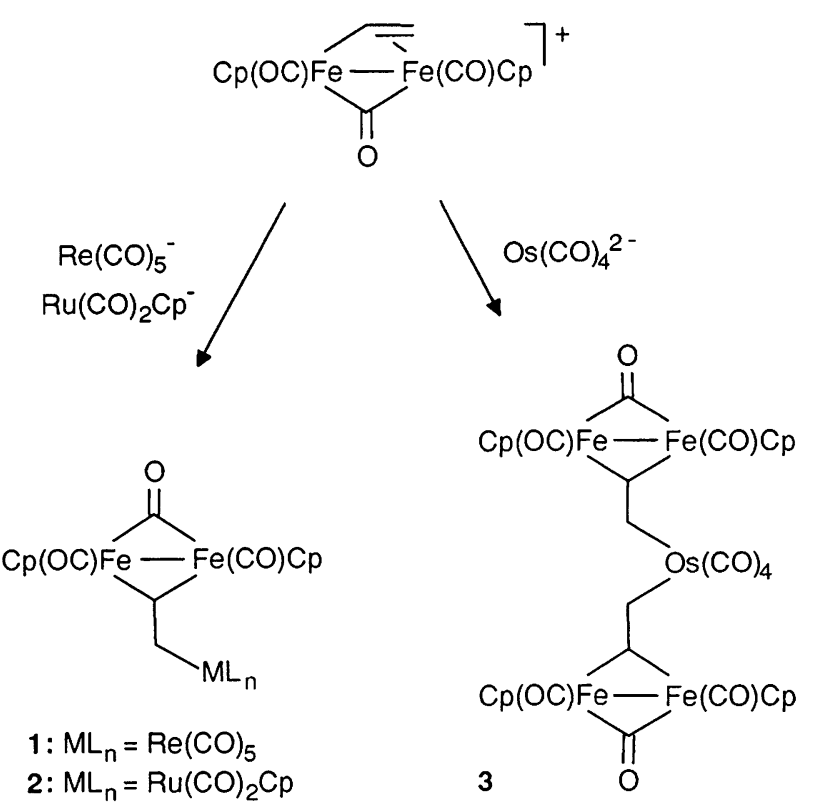

und nichtlineare Optik-Eigenschaften zu erwarten. Auf unerwartete Weise erhielten Fehlhammer und Mitarbeiter eine analoge Verbindung mit einem $\mathrm{N}$-Atom anstelle einer $\mathrm{CH}$ Gruppe als Brücke ${ }^{[10]}$.

Das Verhalten von $\left[\mathrm{Cp} * \mathrm{Ru}\left(\eta^{2}: \eta^{4}-1,3,7 \text {-Octatrien }\right)\right]^{+}(\mathbf{A})$ gegenüber Nucleophilen wurde bisher nicht beschrieben. Dieser Komplex ist als isolierbare Zwischenstufe bei der katalytischen Oligomerisierung von Butadien zu Cyclooctadien von Interesse ${ }^{[11]}$, wobei $[\mathrm{Cp} * \mathrm{Ru} \text { (Butadien) }]^{+}$als katalytische Spezies fungiert. Die Reaktionen der Carbonylmetallate $\left.\left[\operatorname{Re}(\mathrm{CO})_{5}\right)\right]^{-},\left[\mathrm{CpFe}(\mathrm{CO})_{2}\right]^{-}$und $\left[\mathrm{Os}(\mathrm{CO})_{4}\right]^{2-}$ mit A führen unter nucleophiler Addition am terminalen $\mathrm{C}$ Atom der Butadien-Einheit zu den Octadienyl-verbrückten Komplexen 4-6. Diese Verbindungen weisen eine überraschend hohe thermische Stabilität auf. Vor allem für $\mathbf{5}$ ist dies erstaunlich, da sich Alkyl- $\mathrm{Fe}(\mathrm{CO})_{2} \mathrm{Cp}$-Verbindungen sehr leicht unter Bildung von $\left[\mathrm{CpFe}(\mathrm{CO})_{2}\right]_{2}$ zersetzen.

Verwendet man hingegen das Anion $\left[\mathrm{Mn}(\mathrm{CO})_{5}\right]^{-}$, so erhält man das $\mathrm{C}-\mathrm{C}$-Kupplungsprodukt 7 , das auch durch Reduktion mit $\mathrm{Na} / \mathrm{Hg}$ zugänglich ist. Mit $\left[\mathrm{CpM}(\mathrm{CO})_{3}\right]^{-}$ $(\mathrm{M}=\mathrm{Mo}, \mathrm{W})$ und $\mathbf{A}$ bilden sich nur die Salze der Zusammensetzung $\left[\mathrm{Cp} * \mathrm{Ru}\left(\eta^{2}: \eta^{4}-1,3,7-\text { Octatrien }\right)\right]^{+}\left[\mathrm{CpM}(\mathrm{CO})_{3}\right]^{-}$ $(\mathrm{M}=\mathrm{Mo}, \mathrm{W})$. Ähnliche komplexe Salze, wie z.B. [( $\eta^{7}-$ $\left.\left.\mathrm{C}_{7} \mathrm{H}_{7}\right) \mathrm{Mn}\left(\eta^{5}-\mathrm{C}_{5} \mathrm{H}_{4} \mathrm{Me}\right)\right]^{+}\left[\mathrm{CpMo}(\mathrm{CO})_{3}\right]^{-}$oder $\left[\mathrm{Cp}_{2} \mathrm{Co}\right]^{+}$ $\left[\mathrm{Mn}(\mathrm{CO})_{5}\right]^{-}$, sind schon länger bekannt ${ }^{[4]}$. Die Nucleophilie der Anionen $\left[\mathrm{CpM}(\mathrm{CO})_{3}\right]^{-[12,13]}$ reicht hier also nicht für eine Addition aus. Zum anderen ist das Reduktionspotential von $\left[\mathrm{CpM}(\mathrm{CO})_{3}\right]^{-}$größer als das von $\left[\mathrm{Mn}(\mathrm{CO})_{5}\right]^{-[13]}$; so findet auch kein SET-Prozeß statt.

Dienyl- und vor allem Cyclodienyl-Komplexe stellen in der metallorganischen Chemie eine Stoffklasse von großer Vielfalt dar und sind von den meisten Übergangsmetallen beschrieben. Von den kationischen Dienyl-Komplexen sind die des Eisens aufgrund der zahlreichen Anwendungsmöglichkeiten in der organischen Synthese Gegenstand großen Interesses ${ }^{[14]}$. Besonders $\left[(\mathrm{OC})_{3} \mathrm{Fe}\left(\eta^{5}-\mathrm{C}_{6} \mathrm{H}_{7}\right)\right]^{+[15]}$ und analoge Kationen mit substituierten Cyclohexadienyl-Liganden

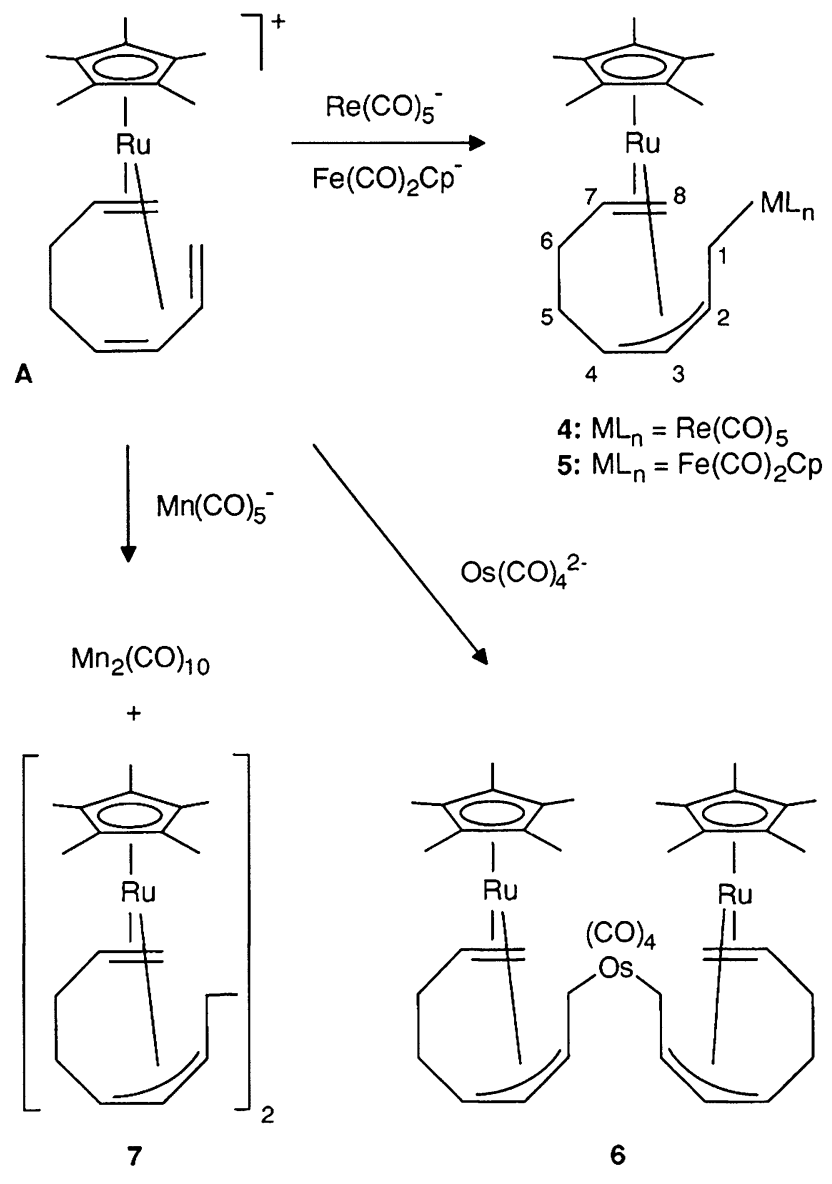

spielen hier eine große Rolle. Die Additionen von Nucleophilen an solche Systeme und die Vielfalt der damit zugänglichen Produkte wird in mehreren Übersichtsartikeln ausführlich behandelt ${ }^{[16]}$. Das Verhalten von $\left[(\mathrm{OC})_{3} \mathrm{M}\left(\eta^{5}\right.\right.$-Dienyl $)]^{+}(\mathrm{M}=\mathrm{Fe}, \mathrm{Ru}$; Dienyl = Hexadienyl, Cyclohexadienyl, Cycloheptadienyl, Bicyclo[5.1.0.]octadienyl) gegenüber Carbonylmetallaten wurde erstmals in unserem Arbeitskreis untersucht ${ }^{[17.18]}$. Bei Reaktionen von $\left[(\mathrm{OC}) \mathrm{Fe}\left(\eta^{4}-\mathrm{C}_{6} \mathrm{H}_{8}\right)\left(\eta^{5}\right.\right.$ $\left.\left.\mathrm{C}_{7} \mathrm{H}_{9}\right)\right]^{+}$mit Hydrid- und Cyanid-Ionen erfolgt ein Angriff am mittleren C-Atom der Dienyl-Gruppe ${ }^{[19]}$. Eine Addition am $\eta^{4}$-Ligand wird mit organischen Nucleophilen nicht beobachtet. Bei $60^{\circ} \mathrm{C}$ erfolgt in Lösung eine rasche Isomerisierung zum thermodynamisch stabileren Bis-1,3-diensystem. Eine Addition am $\eta^{4}$-Dien-Ligand wird mit organischen Nucleophilen nicht beobachtet. Diese ungewöhnliche Regioselektivität wird nur bei nucleophilen Additionen von $\mathrm{PPh}_{3}$ an $\left[(\mathrm{OC})_{3} \mathrm{Mo}\left(\eta^{5}-\mathrm{C}_{7} \mathrm{H}_{9}\right)\right]^{+[20]}$ und von $\mathrm{H}^{-}$oder $\left[(\mathrm{OC})_{3} \mathrm{Cr}\right]_{2}($ Diphenylmethan $\left.)\right]^{-}$an $\left[\mathrm{CpCo}\left(\eta^{5}-\mathrm{C}_{7} \mathrm{H}_{9}\right)\right]^{+}$beschrieben ${ }^{[21,22]}$. Die Carbonylmetallate $\left[\operatorname{Re}(\mathrm{CO})_{5}\right]^{-}$und $\left[\mathrm{Os}(\mathrm{CO})_{4}\right]^{2-}$ addieren sich dagegen am terminalen C-Atom der Cycloheptadienyl-Liganden von $\left[(\mathrm{OC}) \mathrm{Fe}\left(\eta^{4}-\mathrm{Dien}\right)\left(\eta^{5}-\right.\right.$ $\left.\left.\mathrm{C}_{7} \mathrm{H}_{9}\right)\right]^{+}$-Komplexen unter Bildung von 8-10. Bei der Synthese von 8 und 9 wurde jeweils ein zweites, thermisch instabiles Zwischenprodukt mit einem neutralen $\operatorname{Re}(\mathrm{CO})_{5}$-Fragment IR-spektroskopisch nachgewiesen. Wahrscheinlich handelt es sich dabei um das Additionsprodukt von $\left[\operatorname{Re}(\mathrm{CO})_{5}\right]^{-}$am mittleren C-Atom des Dienyl-Liganden. Bei entsprechenden Umsetzungen mit $\left[\mathrm{Mn}(\mathrm{CO})_{5}\right]^{-}$beobachtet 
man wiederum die Bildung des $\mathrm{C}-\mathrm{C}$-Kupplungsproduktes 11.

An $\left[\mathrm{CpCo}\left(\eta^{5}-\mathrm{C}_{6} \mathrm{H}_{7}\right)\right]^{+}$und überraschenderweise ${ }^{[3]}$ auch an $\left[\mathrm{CpCo}\left(\eta^{5}-\mathrm{C}_{7} \mathrm{H}_{9}\right)\right]^{+}$addiert sich $\left[\operatorname{Re}(\mathrm{CO})_{5}\right]^{-}$wie Phosphane ${ }^{[21.23]}$ jeweils am terminalen Kohlenstoff-Atom unter Bildung von 12 und 13. Die homometallischen Redoxprodukte 14,15 sowie $\mathrm{Mn}_{2}(\mathrm{CO})_{10}$ entstehen bei der Umsetzung der entsprechenden Kationen mit $\left[\mathrm{Mn}(\mathrm{CO})_{5}\right]^{-} .15$ wurde schon früher durch elektrochemische Reduktion von $\left[\mathrm{CpCo}\left(\eta^{5}-\mathrm{C}_{7} \mathrm{H}_{9}\right)\right]^{+}$erhalten und durch Röntgenstrukturanalyse charakterisiert ${ }^{[24]}$.
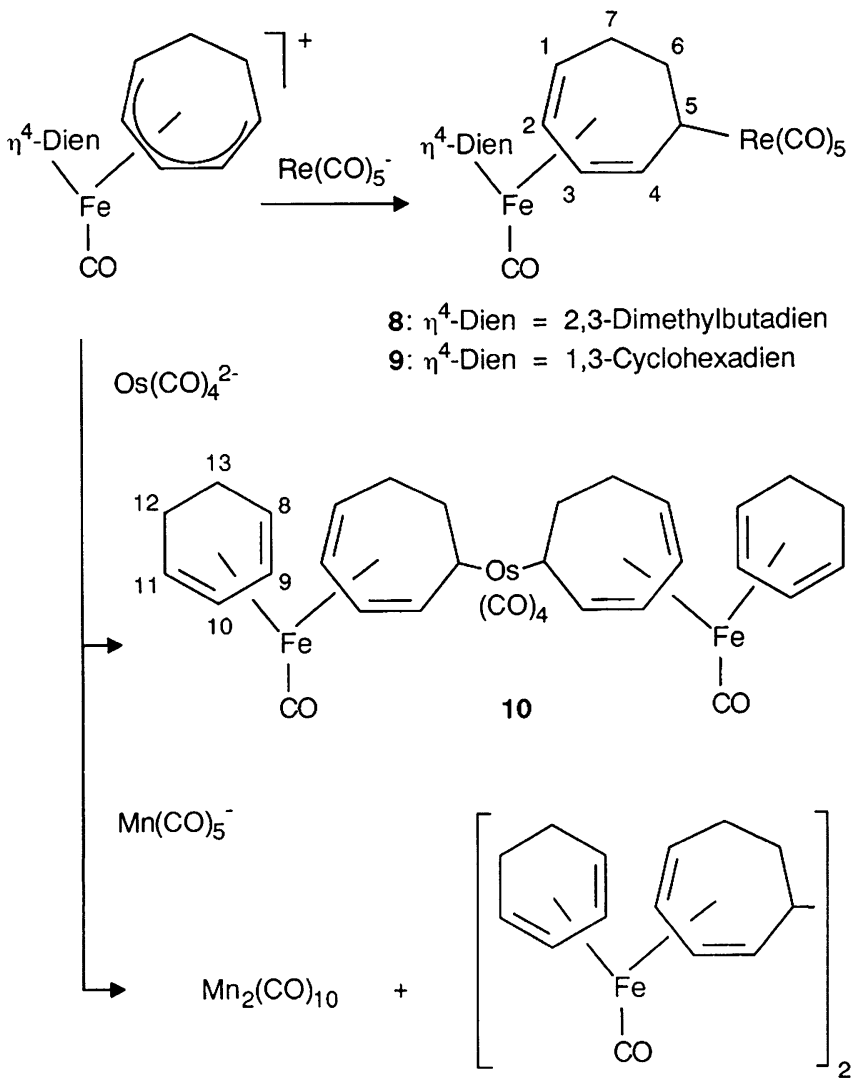

11

In den IR-Spektren von $\mathbf{1 , 4 , 8 , 9 , 1 2}$ und 13 sind die $\mathrm{Ab}$ sorptionen der $\operatorname{Re}(\mathrm{CO})_{5}$-Gruppen, bei 3, $\mathbf{6}$ und $\mathbf{1 0}$ die der $\mathrm{Os}(\mathrm{CO})_{4}$-Gruppe charakteristisch. Insbesondere die $\mathrm{a}_{1}$ Bande des $\operatorname{Re}(\mathrm{CO})_{5}$-Fragments bei $2130 \mathrm{~cm}^{-1}$ und der Os(CO) ${ }_{4}$-Gruppe bei $2100 \mathrm{~cm}^{-1}$ sind gute Sonden für die Bildung der jeweiligen Komplexe. Die CO-Absorptionen der Os $(\mathrm{CO})_{4}$-Komplexe beweisen die schon früher beobachtete cis-Anordnung der beiden Os-C- $\sigma$-Bindungen. Bei 1-4 sind die Banden der verbrückenden CO-Gruppen bei $1744-1779 \mathrm{~cm}^{-1}$ zu erkennen. 8-11 zeigen die Absorptionen der $\mathrm{Fe}-\mathrm{CO}-\mathrm{Gruppen}$ bei $1960 \mathrm{~cm}^{-1}$.

Die ${ }^{1} H$ - und ${ }^{13} C$-NMR-Spektren der Komplexe 1-3 lassen sich durch Vergleich mit bekannten Verbindungen ${ }^{[6.25]}$ genau interpretieren. Neben dem Singulett bei $\delta 4.7$ für die Cyclopentadienyl-Liganden findet man bei $\delta 3.8$ bis 4.4 ein Dublett, welches jeweils der $\mathrm{M}-\mathrm{CH}_{2}$-Gruppe $(\mathrm{M}=\mathrm{Re}, \mathrm{Ru}$, Os) zuzuordnen ist. Typisch für das Signal des H-Atoms der $\mathrm{Fe}-\mathrm{CHR}-\mathrm{Fe}$-Einheit ist eine starke Verschiebung zu
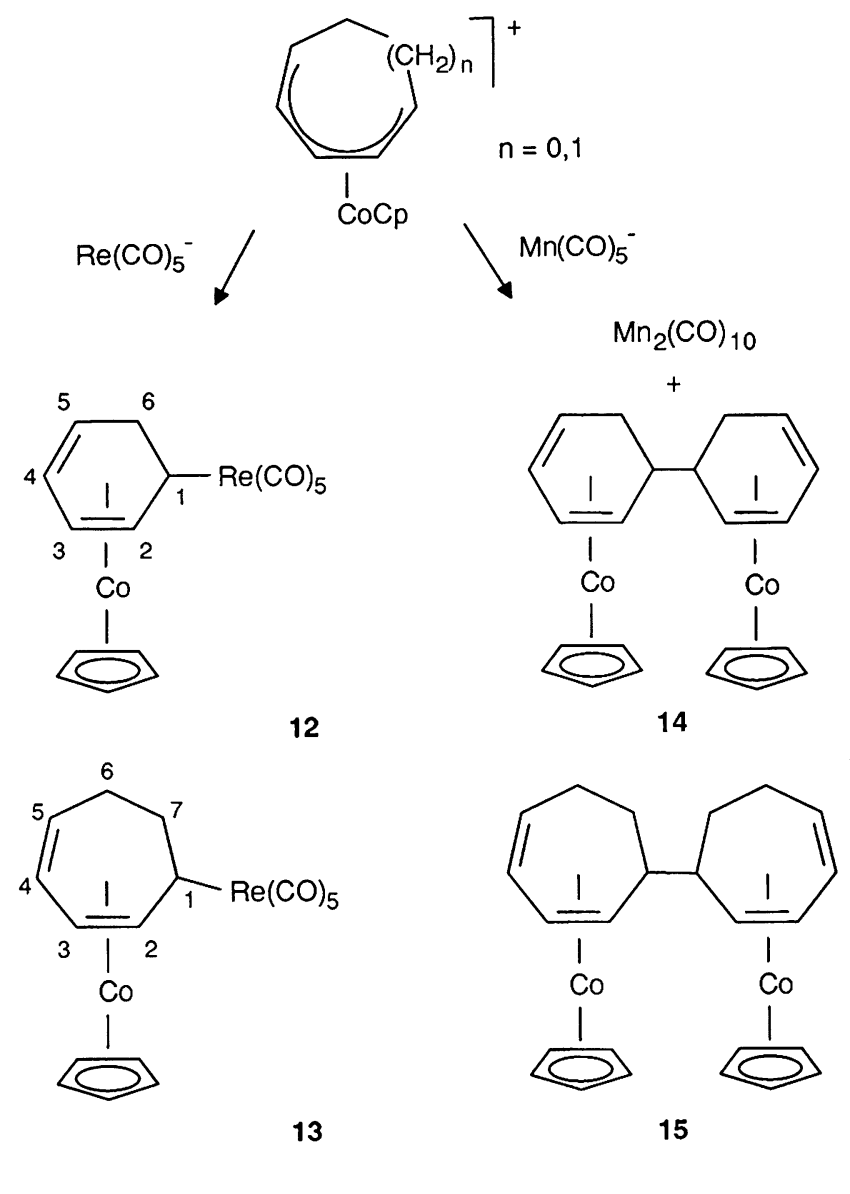

tiefem Feld. Man beobachtet die zugehörigen Tripletts bei $\delta 13$. Wie in den ${ }^{1} \mathrm{H}-\mathrm{NMR}$-Spektren findet man das Signal des Kohlenstoff-Atoms der $\mathrm{M}-\mathrm{CH}_{2} \mathrm{R}$-Gruppe $(\mathrm{M}=\mathrm{Re}$, $\mathrm{Ru}$ ) bei hohem Feld, das der $\mathrm{Fe}-\mathrm{CHR}-\mathrm{Fe}$-Gruppe ist dagegen stark tieffeldverschoben. Für die Kohlenstoff-Atome der endständigen Carbonyl-Liganden sind Verschiebungen um 200 ppm, für die der verbrückenden Carbonyl-Liganden um 275 ppm typisch.

Für 4-15 beweisen die ${ }^{1} \mathrm{H}$ - und ${ }^{13} \mathrm{C}$-NMR-spektroskopischen Daten das Vorliegen der jeweiligen Kohlenwasserstoff-Brücke. Die Interpretation der NMR-Spektren der Verbindung 4 erfolgte mit Hilfe von korrelierten NMRSpektren. Die Daten von 5-7 wurden analog interpretiert. Besonders auffallend sind in den ${ }^{1} \mathrm{H}-\mathrm{NMR}$-Spektren 4-7 die stark hochfeldverschobenen Signale für 5(exo)-H. Dies läßt auf eine ausgeprägte Wannenform der Octadienylbrücke schließen ${ }^{[26]}$. Außerdem fällt auf, daß sich die Kopplungskonstanten der allylischen Protonen stark in Abhängigkeit vom $\sigma$-gebundenen Metall-Fragment unterscheiden. Sie betragen für $\mathrm{ML}_{n}=\operatorname{Re}(\mathrm{CO})_{5} 6.8$ und $9.8 \mathrm{~Hz}$ und für $\mathrm{ML}_{n}=\mathrm{CpFe}(\mathrm{CO})_{2} 3.9$ und $7.8 \mathrm{~Hz}$. In den NMR-Spektren von 6 findet man nur einen Signalsatz. Es ist daher auch in Lösung eine spiegelbildliche Anordnung der beiden $\mathrm{Cp}^{*}$ $\operatorname{Ru}\left(\mu-\eta^{1}: \eta^{1}: \eta^{3}-C_{8} \mathrm{H}_{12}\right)$-Fragmente (siehe Röntgenstrukturanalyse) anzunehmen. Die ${ }^{1} \mathrm{H}-\mathrm{NMR}$-Spektren von 8-15 charakterisieren eindeutig die 1,3-Dien-KohlenwasserstoffBrücken und wurden im Zusammenhang mit bekannten Daten interpretiert ${ }^{[17,21,23,26]}$. 
Durch langsames Abkühlen der gesättigten Pentanlösung auf $-25^{\circ} \mathrm{C}$ erhält man von 4 , durch Überschichten einer gesättigten $\mathrm{CH}_{2} \mathrm{Cl}_{2}$-Lösung mit Pentan von 6 jeweils gelbe Kristalle.

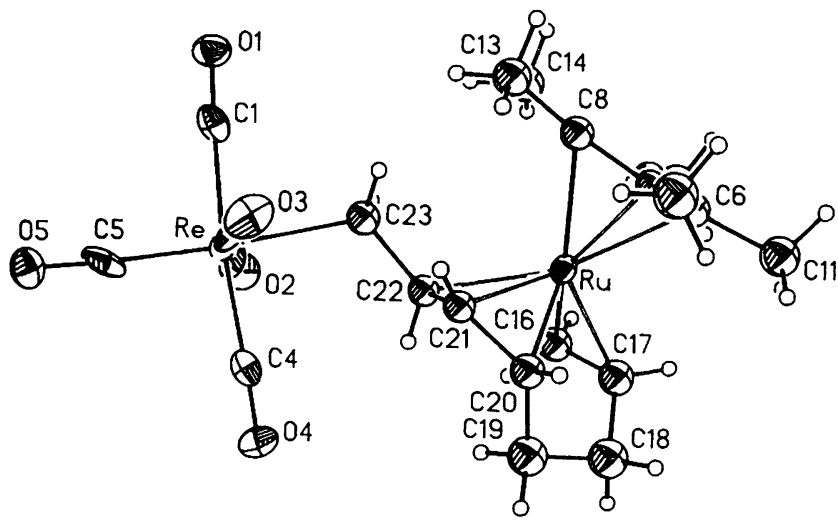

Abb. 1. Molekülstruktur von 4 im Kristall; die thermischen Ellipsoide entsprechen 50\% der Aufenthaltswahrscheinlichkeit; ausgewählte Bindungsabstände $[\mathrm{pm}]$ und -winkel $\left[{ }^{\circ}\right]: \mathrm{Re}-\mathrm{C} 23$ 229.6(18), $\mathrm{Ru}-\mathrm{C} 22$ 223.6(17), $\mathrm{Ru}-\mathrm{C} 21$ 211.1(17), $\mathrm{Ru}-\mathrm{C} 20$ 216.3(17), $\mathrm{Ru}-\mathrm{C} 17$ 217.2(19), $\mathrm{Ru}-\mathrm{C} 16 \quad 216.2(18), \quad \mathrm{C} 16-\mathrm{C} 17$ 137.2(25), $\mathrm{C} 17-\mathrm{C} 18$ 149.6(28), C19-C20 156.0(27), C20-C21 141.5(24), C21-C22 141.2(21); $\mathrm{Re}-\mathrm{C} 23-\mathrm{C} 22$ 116.5(12), $\mathrm{Ru}-\mathrm{C} 16-\mathrm{C} 17$ 71.1(11), Ru$\mathrm{C} 20-\mathrm{C} 21$ 68.7(9), Ru-C21-C22 75.9(10), C20-Ru-C22 68.1(6), $\mathrm{C} 21-\mathrm{C} 22-\mathrm{C} 23120.8(14), \mathrm{C} 20-\mathrm{C} 21-\mathrm{C} 22$ 121.2(15), $\mathrm{C} 19-\mathrm{C} 20-$ C21 122.4(15), C17-C18-C19 108.6(16), C18-C19-C20 108.5(17)

Die Röntgenstrukturanalysen von 4 (Abb.1) und 6 (Abb.2) bestätigen die Koordination der $\mathrm{Cp} * \mathrm{Ru}$-Einheit an eine Allyl- und eine Alkengruppe des Octadienylliganden. Deutlich ist auch die Wannenform der Kohlenwasserstoff-Brükken zu erkennen. Das $\operatorname{Re}(\mathrm{CO})_{5}$ - bzw. Os $(\mathrm{CO})_{4}$-Fragment ist immer in exo-Stellung gegenüber der $\mathrm{RuCp}^{*}$-Gruppe angeordnet. Die Re-C- bzw. Os-C-Bindungen in 4, 6 und 9 sind ähnlich lang wie in vergleichbaren Komplexen mit $\mathrm{M}-\mathrm{C}-\sigma-\mathrm{Bindung}{ }^{[2,17,18,27]}$. Die C-C-Bindungsabstände in 4 und 6 sind im Allyl-Fragment anders als bei CpMo$(\mathrm{CO})_{2}\left(\mu-\eta^{1}: \eta^{3}-\mathrm{C}_{5} \mathrm{H}_{8}\right) \operatorname{Re}(\mathrm{CO})_{5}{ }^{[28]}$ fast identisch. Die Winkel an den allylischen C-Atomen kommen jeweils dem Idealwert von $120^{\circ}$ sehr nahe. Die Abstände der terminalen CAtome der Allyl-Systeme zum Ruthenium-Atom in $\mathbf{4}$ und $\mathbf{6}$ unterscheiden sich um etwa $7 \mathrm{pm}$. Mit 137.2(25) pm bei 4 und $139.0(1)$ pm bei 6 ist der C-C-Bindungsabstand in der $\pi$-gebundenen Alken-Einheit etwas länger als in einem unkoordinierten Alken. Bei $\mathrm{Cp} * \operatorname{Ir}\left(\eta^{2}-\mathrm{C}_{2} \mathrm{H}_{4}\right)\left(\mu-\eta^{1}: \eta^{2}-\right.$ $\left.\mathrm{C}_{3} \mathrm{H}_{5}\right) \operatorname{Re}(\mathrm{CO})_{5}{ }^{[1]}$ ist der Metallacyclopropan-Charakter stärker ausgeprägt; der entsprechende Abstand beträgt dort $145 \mathrm{pm}$.

Im Gegensatz zu anderen $\left(\mathrm{L}_{n} \mathrm{M}-\mathrm{KW}\right)_{2} \mathrm{Os}(\mathrm{CO})_{4}$-Komplexen ${ }^{[1,2,18,27]}$ mit Chiralitätszentren am Metall $\sigma$-gebundenen C-Atom beweist die Röntgenstrukturanalyse von $\mathbf{6}$ eine $C_{s}$-Symmetrie. Für eine Röntgenstrukturanalyse (Abb.3) geeignete Kristalle von 9 konnten durch langsames Abkühlen einer gesättigten Pentanlösung auf $-30^{\circ} \mathrm{C}$ erhalten werden.

Die beiden Kohlenwasserstoff-Liganden in 9 sind jeweils über zwei konjugierte Doppelbindungen an das zentrale Eisen-Atom gebunden und liegen in einer Wannenkonformation vor. Der Abstand $\mathrm{Fe}-\mathrm{C} 7$ mit benachbarter $\mathrm{Re}(\mathrm{CO})_{5^{-}}$

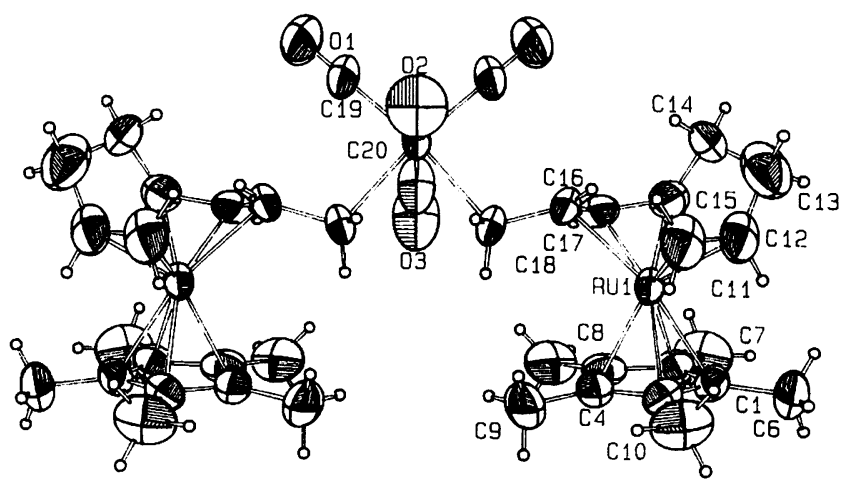

Abb. 2. Molekülstruktur von 6 im Kristall: die thermischen Ellipsoide entsprechen 50\% der Aufenthaltswahrscheinlichkeit; ausgewählte Bindungsabstände [pm] und -winkel $\left[{ }^{\circ}\right]$ : Os-C18 223.4(7), Os-C21 195.0(1), Os-C20 192.0(1), Ru-C11 219.7(7), Ru-C12 219.1(7), $\mathrm{Ru}-\mathrm{C} 15$ 216.8(7), Ru-C16 210.7(6), Ru-C17 223.6(6), C11-C12 $139.0(1), \quad \mathrm{C} 12-\mathrm{C} 13 \quad 148.0(1), \quad \mathrm{C} 14-\mathrm{C} 15 \quad 158.0(1), \quad \mathrm{C} 15-\mathrm{C} 16$ 140.0(1), C16-C17 141.2(9), C17-C18 148.0(9); C18-Os-C18' 81.2(2), C18-Os-C19 172.9(3), C18-Os-C20 85.7(3), $\mathrm{Ru}-\mathrm{C} 11-\mathrm{C} 12$ 71.3(4), Os-C18-C17 115.8(5), C11-C12-C13 $124.8(8), \quad \mathrm{C} 12-\mathrm{C} 13-\mathrm{C} 14 \quad 110.3(7), \mathrm{C} 13-\mathrm{C} 14-\mathrm{C} 15$ 112.6(7), $\mathrm{C} 14-\mathrm{C} 15-\mathrm{C} 16$ 121.4(7), C16-C17-C18 122.1(6)

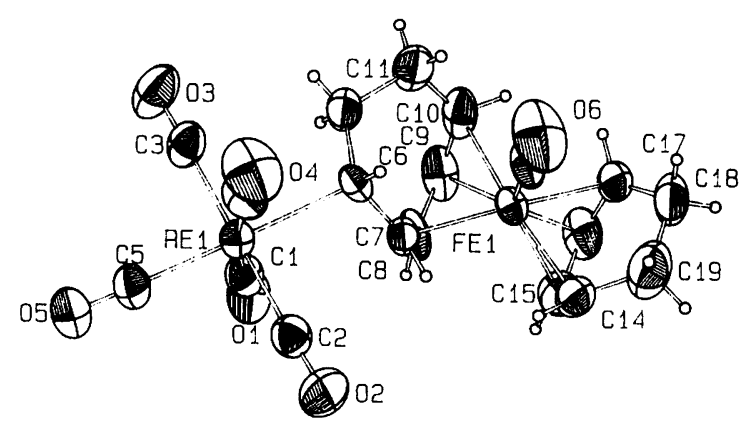

Abb. 3. Molekülstruktur von 9 im Kristall: die thermischen Ellipsoide entsprechen 50\% der Aufenthaltswahrscheinlichkeit; ausgewählte Bindungsabstände $[\mathrm{pm}]$ und -winkel $\left[^{\circ}\right]: \mathrm{Re}-\mathrm{C} 6$ 234.6(5), $\mathrm{Fe}-\mathrm{C} 7$ 218.1(5), $\mathrm{Fe}-\mathrm{C} 10$ 211.3(7), $\mathrm{Fe}-\mathrm{C} 8$ 203.6(6), $\mathrm{Fe}-\mathrm{C} 17$ 212.1(6), $\mathrm{Fe}-\mathrm{C} 9$ 204.1(7), $\mathrm{Fe}-\mathrm{C} 16$ 204.4(7), $\mathrm{Fe}-\mathrm{C} 13$ 174.4(6), C7-C 142.3(8), C8-C9 143.3(9), C9-C10 139.0(1), C14-C15 138.0(1), C15-C16 138.0(1), C16-C17 138.0(1), C6-C7 148.1(8), C10-C11 152(1), C14-C19 151.0(1), C13-O6 114(8); C5-Re-C6 178.2(2), $\mathrm{C} 2-\mathrm{Re}-\mathrm{C} 6$ 89.4(2), C13-Fe-C15 127.0(3), C13-Fe-C14 89.1(3), $\mathrm{C} 7-\mathrm{Fe}-\mathrm{C} 14 \quad 101.5(2), \quad \mathrm{C} 8-\mathrm{Fe}-\mathrm{C} 15 \quad 93.6(3), \quad \mathrm{C} 17-\mathrm{C} 18-\mathrm{C} 19$ 109.9(6), $\mathrm{C} 16-\mathrm{C} 17-\mathrm{C} 18$ 120.7(7), C6-C $7-\mathrm{C} 8$ 125.6(5), $\mathrm{C} 9-\mathrm{C} 10-\mathrm{C} 11$ 126.4(6), C6-C12-C11 115.6(6), C14-C.19-C18 $111.5(6)$

Gruppe ist mit 218.1(5) pm relativ groß. Die beiden aus den jeweils vier $\mathrm{sp}^{2}$-hybridisierten C-Atomen gebildeten Ebenen liegen annähernd parallel. Diese Geometrie wurde auch bei anderen Verbindungen mit zwei $\eta^{4}$-Dien-Liganden beobachtet ${ }^{[29]}$.

Die heterometallischen, Kohlenwasserstoff-verbrückten Komplexe erscheinen als Vorläufer von heterogenen Katalysatoren von Interesse. Mit ihnen kann die Abscheidung von Metallatomen auf Trägern in bestimmter Stöchiometrie sowie in durch die Kohlenwasserstoffbrücken vorgegebenen Abstand erfolgen. In orientierenden Versuchen wurden die Komplexe 5-7 aus einer Hexan- oder Benzollösung auf $\gamma$ $\mathrm{Al}_{2} \mathrm{O}_{3}\left(112 \mathrm{~m}^{2} / \mathrm{g}\right.$, Merck) abgeschieden und anschließend bei $500^{\circ} \mathrm{C} \mathrm{im} \mathrm{H}_{2}$-Strom zersetzt oder reduziert. Bei der Gasphasenhydrierung von Benzol erwies sich z. B. der aus 6 syn- 
thetisierte Katalysator als wesentlich aktiver als der aus einem 1:2-Gemisch von $\mathrm{Ru}_{3}(\mathrm{CO})_{12}$ und $\mathrm{Os}_{3}(\mathrm{CO})_{12}$ erhaltene Katalysator.

Der Deutschen Forschungsgemeinschaft, dem Fonds der Chemischen Industrie, der DEGUSSA AG, Frankfurt, sowie der Wacker Chemie $G m b H$, München, gilt unser herzlicher Dank für großzügige Förderung. Herrn Prof. Dr. H.-P. Boehm, München, danken wir für die Bereitstellung von Geräten.

\section{Experimenteller Teil}

Alle Reaktionen wurden unter Argon mit sorgfältig getrockneten Lösungsmitteln durchgeführt. - Zersetzungspunkte (nicht korrigiert): Schmelzpunktapparat der Firma Büchi (Typ 5). - IR: Perkin-Elmer 841. - NMR: Jeol FX 90 Q, Jeol GSX 270 und Jeol EX 400. - Elementaranalysen: Heraeus VT, CHN-O-Rapid. Röntgenstrukturanalysen: Tab. 1.

Tab. 1. Kristallographische Daten von 4, 6 und $9^{[34]}{ }^{*}$ H-Atome an C11 bis C15 nicht verfeinert (rigid model, $U_{\mathrm{H}}=1000 \mathrm{pm}^{2}$ ), restliche H-Atome verfeinert (rigid model, $U_{\mathrm{H}}=1.2 \mathrm{U}_{\mathrm{c}}$ )

\begin{tabular}{|c|c|c|c|}
\hline & 4 & 6 & 9 \\
\hline Summenformel & $\mathrm{C}_{23} \mathrm{H}_{27} \mathrm{O}_{5} \mathrm{ReRu}$ & $\mathrm{C}_{40} \mathrm{H}_{54} \mathrm{O}_{4} \mathrm{OsRu}_{2}$ & $\mathrm{C}_{19} \mathrm{H}_{17} \mathrm{O}_{6} \mathrm{FeRe}$ \\
\hline Molmasse & 670.7 & 991.21 & 583.99 \\
\hline Kristallgröße & $0.43 \times 0.2 \times 0.1$ & $0.2 \times 0.33 \times 0.47$ & $0.1 \times 0.3 \times 0.53$ \\
\hline Kristallsystem & monoklin & orthorhombisch & monoklin \\
\hline Raumgruppe & $\mathrm{P} 2{ }_{1} / \mathrm{c}$ & Pnma & $\mathrm{P} 2{ }_{1} / \mathrm{c}$ \\
\hline $\mathrm{a}[\mathrm{pm}]$ & $1118.0(5)$ & $1044.2(3)$ & $1156.9(2)$ \\
\hline $\mathrm{b}[\mathrm{pm}]$ & $1729.5(7)$ & $3178.8(6)$ & $1377.0(4)$ \\
\hline $\mathrm{c}[\mathrm{pm}]$ & $1243.6(5)$ & $1163.6(4)$ & $1230.4(3)$ \\
\hline$\beta\left[^{\circ}\right]$ & $99.39(3)$ & - & $103.30(2)$ \\
\hline Volumen $\left[\mathrm{nm}^{3}\right]$ & $2.372(2)$ & 3.8622 & 1.9076 \\
\hline $\mathrm{Z}$ & 4 & 4 & 4 \\
\hline Dichte (ber.) $\left[\mathrm{g} / \mathrm{cm}^{3}\right]$ & 1.878 & 1.705 & 2.031 \\
\hline Abs.-koeff. $\left[\mathrm{mm}^{-1}\right]$ & 5.821 & 4.081 & 7.22 \\
\hline Diffraktometer & Syntex-Nicolet R3 & E.-N. CAD4 & E.-N. CAD4 \\
\hline Meßtemperatur $\left[{ }^{\circ} \mathrm{C}\right]$ & 18 & 23 & $23 \pm 1$ \\
\hline Meßbereich $\left.\left.2 \Theta\right|^{\circ}\right]$ & $4-40$ & $4-46$ & $4-46$ \\
\hline Scanbreite $\left[{ }^{\circ}\right]$ & 1.0 & $0.60+0.35 \operatorname{Tan}(\theta)$ & $0.80+0.35 \operatorname{Tan}(\theta)$ \\
\hline Meßzeit & $3-10 \% / \mathrm{min}$ & $60 \mathrm{~s}(\max )$ & $60 \mathrm{~s}(\max )$ \\
\hline Gemessene Reflexe & 2537 & 3069 & 2930 \\
\hline Symm. unabh. Reflexe & 2227 & 2726 & 2764 \\
\hline Beobachtete Reflexe & $1812[\mathrm{~F}>3 \sigma(\mathrm{F})]$ & $2358[\mathrm{I}>3 \mathrm{o}(\mathrm{I})]$ & $2364[\mathrm{I}>2 \sigma(\mathrm{I})]$ \\
\hline hkl-Bereich & $\begin{array}{l}0 \leq \mathrm{h} \leq 11 \\
0 \leq \mathrm{k} \leq 17 \\
-12 \leq \mathrm{l} \leq 12\end{array}$ & $+h+k+l$ & - \\
\hline Programm & SHELXTL PLUS 3.43 & SHELXTL PLUS 3.43 & SHELXTL PLUS 3.43 \\
\hline H-Atome & geom. pos.* & geom. pos. & geom. pos. \\
\hline Verfeinerte Parameter & 182 & 220 & 244 \\
\hline Gewichtung & $w=1 / \sigma^{2}(F)+10^{-4}(F)^{2}$ & $w=1 / \sigma^{2}(F)$ & $w=1 / \sigma^{2}(F)$ \\
\hline $\mathrm{R}$ & 0.0495 & 0.0329 & 0.0256 \\
\hline $\mathrm{R}_{\mathrm{w}}$ & 0.0476 & 0.0429 & 0.0357 \\
\hline Extrema der letzten & $2.60 /-0.67$ & $1.156 /-1.524$ & $0.53 /-1.237$ \\
\hline \multicolumn{4}{|c|}{$\begin{array}{l}\text { Differenz-Fourier-Synthese } \\
{\left[\mathrm{e} \times 10^{-6} \mathrm{pm}^{-3}\right]}\end{array}$} \\
\hline $\begin{array}{l}\text { Absorptionskorrektur } \\
\text { (min./max. Transmission) }\end{array}$ & $\begin{array}{l}0.0698 / 0.1015 \\
\text { empirisch }\end{array}$ & $\begin{array}{l}0.7615 / 0.9969 \\
\text { empirisch }\end{array}$ & $\begin{array}{l}0.4604 / 0.9984 \\
\text { empirisch }\end{array}$ \\
\hline
\end{tabular}

Die Ausgangskomplexe $\mathrm{Na}\left[\mathrm{Mn}(\mathrm{CO})_{5}\right]^{[30]}, \quad \mathrm{Na}\left[\mathrm{Re}(\mathrm{CO})_{5}\right]^{[31]}$, $\mathrm{Na}\left[\mathrm{CpFe}(\mathrm{OC})_{2}\right]^{[32]}, \quad \mathrm{Na}_{2}\left[\mathrm{Os}(\mathrm{CO})_{4}\right]^{[33]}, \quad\left[\mathrm{Cp}_{2} \mathrm{Fe}_{2}(\mathrm{CO})_{2}(\mu-\mathrm{CO})(\mu-\right.$ $\left.\left.\eta^{\prime}: \eta^{2}-\mathrm{CH}=\mathrm{CH}_{2}\right)\right] \mathrm{BF}_{4}{ }^{[6]},\left[\mathrm{Cp} * \mathrm{Ru}\left(\eta^{2}: \eta^{4}-\mathrm{C}_{8} \mathrm{H}_{12}\right)\right] \mathrm{SO}_{3} \mathrm{CF}_{3}{ }^{[1]},[(\mathrm{OC})-$ $\mathrm{Fe}\left(\eta^{4}\right.$-Dien $\left.)\left(\eta^{5}-\mathrm{C}_{7} \mathrm{H}_{9}\right)\right] \mathrm{BF}_{4}$ (Dien = 2,3-Dimethylbutadien, 1,3-Hexadien $)^{[19]},\left[\mathrm{CpCo}\left(\eta^{5}-\mathrm{C}_{6} \mathrm{H}_{7}\right)\right] \mathrm{PF}_{6},\left[\mathrm{CpCo}\left(\eta^{5}-\mathrm{C}_{7} \mathrm{H}_{9}\right)\right] \mathrm{PF}_{6}{ }^{[21.35]}$ wurden nach Literaturangaben dargestellt.

$\mathrm{Cp}_{2} \mathrm{Fe}_{2}(\mathrm{CO})_{2}(\mu-\mathrm{CO})\left[\mu-\mathrm{CHCH}_{2}-\mathrm{Re}(\mathrm{CO})_{5}\right]$ (1): Aus $200 \mathrm{mg}$ $(0.31 \mathrm{mmol}) \mathrm{Re}_{2}(\mathrm{CO})_{10}$ wird in $15 \mathrm{ml}$ THF durch Reduktion mit
$\mathrm{Na} / \mathrm{Hg}$ eine gelborangefarbene $\mathrm{Na}\left[\operatorname{Re}(\mathrm{CO})_{5}\right]$-Lösung hergestellt. Diese wird auf $-70^{\circ} \mathrm{C}$ gekühlt und portionsweise mit $163 \mathrm{mg}(0.37$ mmol) $\left[\mathrm{Cp}_{2} \mathrm{Fe}_{2}(\mathrm{CO})_{2}(\mu-\mathrm{CO})\left(\mu-\eta^{1}: \eta^{2}-\mathrm{CH}=\mathrm{CH}_{2}\right)\right] \mathrm{BF}_{4}$ versetzt. Es bildet sich rasch eine dunkelrote Lösung. Nach etwa $15 \mathrm{~min}$. Rühren wird langsam auf Raumtemp. erwärmt und anschließend das Lösungsmittel i. Hochvak. entfernt. Das Rohprodukt wird dreimal mit je $10 \mathrm{ml}$ Pentan gewaschen, um in geringen Mengen vorhandenes $\operatorname{Re}_{2}(\mathrm{CO})_{10} \mathrm{zu}$ entfernen. Zur weiteren Reinigung wird der Rückstand zweimal mit $10 \mathrm{ml}$ Ether extrahiert, der Extrakt über Kieselgel filtriert, die Lösung auf etwa $3 \mathrm{ml}$ eingeengt und das Produkt durch Zugabe von Pentan ausgefällt. 1 fällt nach Waschen mit Pentan und Trocknen i. Hochvak. $(6 \mathrm{~h})$ als dunkelrotes Pulver an. Ausb. $165 \mathrm{mg}(64 \%)$; Zers.-P. $120^{\circ} \mathrm{C}$. - IR (Nujol): $\tilde{v}=2119$ $\mathrm{cm}^{-1}$ w, 1994 vs, $1960 \mathrm{~s}, 1918 \mathrm{~m}, 1761 \mathrm{~m} .-{ }^{1} \mathrm{H}-\mathrm{NMR}(90 \mathrm{MHz}$, $\left.\mathrm{CDCl}_{3}\right): \delta=3.81\left(\mathrm{~d}, 2 \mathrm{H}, \mathrm{ReCH}_{2} \mathrm{R},{ }^{3} J=8.8 \mathrm{~Hz}\right), 4.66(\mathrm{~s}, 10 \mathrm{H}$, Cp), 13.07 (t, $\left.1 \mathrm{H}, \mathrm{FeCHR}-\mathrm{Fe},{ }^{3} J=8.8 \mathrm{~Hz}\right) .-{ }^{13} \mathrm{C}-\mathrm{NMR}(100.5$ $\left.\mathrm{MHz}, \quad \mathrm{CD}_{2} \mathrm{Cl}_{2}\right): \delta=33.10\left(\mathrm{ReCH}_{2} \mathrm{R}\right), 87.84(\mathrm{Cp}), 181.65$ $\left(\mathrm{ReCO}_{\mathrm{ax}}\right), \quad 187.60 \quad\left(\mathrm{ReCO}_{\text {ӓq }}\right), 200.15(\mathrm{Fe}-\mathrm{CHR}-\mathrm{Fe}), 213.73$ (FeCO), 274.85 ( $\mathrm{Fe}-\mathrm{CO}-\mathrm{Fe}$ ). $-\mathrm{C}_{20} \mathrm{H}_{13} \mathrm{Fe}_{2} \mathrm{O}_{8} \mathrm{Re}$ (679.1): ber. $\mathrm{C}$ 35.37, H 1.93; gef. C 33.76, H 2.48.

$\mathrm{Cp}_{2} \mathrm{Fe}_{2}(\mathrm{CO})_{2}(\mu-\mathrm{CO})\left[\mu-\mathrm{CHCH}_{2}-\mathrm{Ru}(\mathrm{CO})_{2} \mathrm{Cp}\right]$ (2): Aus $100 \mathrm{mg}$ $(0.23 \mathrm{mmol})\left[\mathrm{CpRu}(\mathrm{CO})_{2}\right]_{2}$ wird in $10 \mathrm{ml} \mathrm{THF}$ durch Reduktion mit $\mathrm{Na} / \mathrm{Hg}$ eine rotorangefarbene $\mathrm{Na}\left[\mathrm{CpRu}(\mathrm{CO})_{2}\right]$-Lösung hergestellt. Diese wird auf $-70^{\circ} \mathrm{C}$ gekühlt und portionsweise mit $158 \mathrm{mg}$ $(0.36 \mathrm{mmol})\left[\mathrm{Cp}_{2} \mathrm{Fe}_{2}(\mathrm{CO})_{2}(\mu-\mathrm{CO})\left(\mu-\eta^{1}: \eta^{2}-\mathrm{CH}=\mathrm{CH}_{2}\right)\right] \mathrm{BF} \mathrm{B}_{4}$ versetzt. Es bildet sich rasch eine dunkelrote Lösung. Nach etwa $15 \mathrm{~min}$. Rühren wird langsam auf Raumtemp. erwärmt und anschließend das Lösungsmittel i. Hochvak. entfernt. Das Rohprodukt wird zweimal mit je $10 \mathrm{ml}$ Pentan gewaschen, um in geringen Mengen vorhandenes $\left[\mathrm{CpRu}(\mathrm{CO})_{2}\right]_{2} \mathrm{zu}$ entfernen. Zur weiteren Reinigung wird der Rückstand viermal mit $10 \mathrm{ml}$ Ether extrahiert, die Lösung auf etwa $3 \mathrm{ml}$ eingeengt und das Produkt durch Zugabe von Pentan ausgefällt. Man erhält 2 nach Waschen mit Pentan und Trocknen i. Hochvak. (6 h) als rotviolettes Pulver. Ausb. $101 \mathrm{mg}$ (49\%); Zers.P. $151^{\circ} \mathrm{C}$. - IR (Nujol): $\tilde{v}=1994 \mathrm{~cm}^{-1} \mathrm{~s}, 1949 \mathrm{vs}, 1929 \mathrm{~s}, 1918 \mathrm{~m}$, 1779 s. $-{ }^{\prime} \mathrm{H}-\mathrm{NMR}\left(90 \mathrm{MHz}, \mathrm{CD}_{2} \mathrm{Cl}_{2}\right): \delta=4.36\left(\mathrm{~d}, 2 \mathrm{H}, \mathrm{RuCH}_{2} \mathrm{R}\right.$, $\left.{ }^{3} J=8.8 \mathrm{~Hz}\right), 4.71(\mathrm{~s}, 10 \mathrm{H}, \mathrm{FeCp}), 5.44(5 \mathrm{H}, \mathrm{RuCp}), 12.50(\mathrm{tr}, 1 \mathrm{H}$, $\left.\mathrm{Fe}-\mathrm{CHR}-\mathrm{Fe},{ }^{3} \mathrm{~J}=8.8 \mathrm{~Hz}\right) . \quad{ }^{13} \mathrm{C}-\mathrm{NMR}\left(22.5 \mathrm{MHz}, \mathrm{CDCl}_{3}\right)$ : $\delta=33.28\left(\mathrm{RuCH}_{2} \mathrm{R}\right), 87.85(\mathrm{FeCp}), 89.42(\mathrm{RuCp}), 197.71(\mathrm{RuCO})$, 203.84 (Fe-CHR-Fe), 213.59 (FeCO), 274.67 ( $\mathrm{Fe}-\mathrm{CO}-\mathrm{Fe})$. $\mathrm{C}_{22} \mathrm{H}_{18} \mathrm{Fe}_{2} \mathrm{O}_{5} \mathrm{Ru}(574.3)$ : ber. C 46.01, H 3.16; gef. C 44.65, H 3.61.

$\left\{\mathrm{Cp}_{2} \mathrm{Fe}_{2}(\mathrm{CO})_{2}(\mu-\mathrm{CO})\left(\mu-\mathrm{CHCH}_{2}-\right\}_{2} \mathrm{Os}(\mathrm{CO})_{4}\right.$ (3): Eine Suspension von $50 \mathrm{mg}(0.14 \mathrm{mmol}) \mathrm{Na}_{2}\left[\mathrm{Os}(\mathrm{CO})_{4}\right]$ in $10 \mathrm{ml} \mathrm{THF}$ wird auf $-70^{\circ} \mathrm{C}$ abgekühlt und mit $123 \mathrm{mg}(0.28 \mathrm{mmol})\left[\mathrm{Cp}_{2} \mathrm{Fe}_{2^{-}}\right.$ $\left.(\mathrm{CO})_{2}(\mu-\mathrm{CO})\left(\mu-\eta^{\prime}: \eta^{2}-\mathrm{CH}=\mathrm{CH}_{2}\right)\right] \mathrm{BF}_{4}$ versetzt. Die rote Suspension wird etwa $1 \mathrm{~h}$ gerührt, langsam auf Raumtemp. erwärmt und anschließend das Lösungsmittel i. Hochvak. entfernt. Der Rückstand

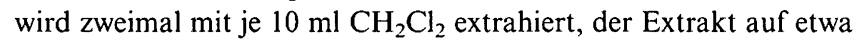
$4 \mathrm{ml}$ eingeengt und auf eine Chromatographiesäule (Durchmesser $1 \mathrm{~cm}, 20 \mathrm{~cm}$ lang, Kieselgel, $\mathrm{CH}_{2} \mathrm{Cl}_{2}$ ) gegeben. $\mathrm{Mit} \mathrm{CH}_{2} \mathrm{Cl}_{2}$ eluiert man nacheinander eine gelbe, eine orangefarbene und eine breite rote Bande. Letztere Fraktion enthält das Produkt und wird vom Lösungsmittel befreit. Danach wird 3 mit Pentan gewaschen und 6 h i. Hochvak. getrocknet. Ausb. $68 \mathrm{mg}(48 \%)$; Zers.-P. $128^{\circ} \mathrm{C}$. - IR (Nujol): $\tilde{v}=2119 \mathrm{~cm}^{-1} \mathrm{vw}, 2047 \mathrm{~m}, 2039 \mathrm{~m}, 2016 \mathrm{~s}, 2008 \mathrm{w}, 1956$ vs, $1925 \mathrm{~m}, 1751$ s. $-{ }^{1} \mathrm{H}-\mathrm{NMR}\left(90 \mathrm{MHz}, \mathrm{CD}_{2} \mathrm{Cl}_{2}\right): 4.01$ (d, $4 \mathrm{H}$, $\left.\mathrm{OsCH}_{2} \mathrm{R},{ }^{3} \mathrm{~J}=8.4 \mathrm{~Hz}\right), 4.72(\mathrm{~s}, 20 \mathrm{H}, \mathrm{Cp}), 13.01(\mathrm{t}, 2 \mathrm{H}$, $\mathrm{Fe}-\mathrm{CHR}-\mathrm{Fe},{ }^{3} \mathrm{~J}=8.4 \mathrm{~Hz}$ ). - Wegen der geringen Löslichkeit von 3 in den gängigen Lösungsmitteln konnte kein ${ }^{13} \mathrm{C}$-NMR-Spektrum gemessen werden. $-\mathrm{C}_{34} \mathrm{H}_{26} \mathrm{Fe}_{4} \mathrm{O}_{10} \mathrm{Os}$ (1008.1): ber. $\mathrm{C} 40.51$, H 2.61; gef. C 41.07, H 3.04 .

Umsetzung von $\left[\mathrm{Cp}_{2} \mathrm{Fe}_{2}(\mathrm{CO})_{2}(\mu-\mathrm{CO})\left(\mu-\mathrm{CHCH}_{2}-\right)\right] \mathrm{BF}_{4}$ mit $\left[\mathrm{Mn}(\mathrm{CO})_{5}\right]^{-},\left[\mathrm{CpFe}(\mathrm{CO})_{2}\right]^{-},\left[\mathrm{CpW}(\mathrm{CO})_{3}\right]^{-}$und Natriumamalgam 
a) Aus $100 \mathrm{mg}(0.26 \mathrm{mmol}) \mathrm{Mn}_{2}(\mathrm{CO})_{10}$ wird in $15 \mathrm{ml} \mathrm{THF}$ durch Reduktion mit Natriumamalgam eine gelbgrüne $\mathrm{Na}\left[\mathrm{Mn}(\mathrm{CO})_{5}\right]-$ Lösung hergestellt. Diese wird auf $-70^{\circ} \mathrm{C}$ gekühlt und portionsweise mit $183 \mathrm{mg}(0.42 \mathrm{mmol})\left[\mathrm{Cp}_{2} \mathrm{Fe}_{2}(\mathrm{CO})_{2}(\mu-\mathrm{CO})\left(\mu-\eta^{1}: \eta^{2}\right.\right.$ $\left.\mathrm{CH}=\mathrm{CH}_{2}\right) \mathrm{BF}_{4}$ versetzt. Innerhalb weniger min bildet sich eine Suspension mit einem ziegelroten Festkörper. Man erwärmt langsam auf Raumtemp., zentrifugiert und pipettiert die überstehende THF-Lösung ab, die $\mathrm{Mn}_{2}(\mathrm{CO})_{10}$ enthält. Das Rohprodukt wird zur Reinigung zweimal mit je $10 \mathrm{ml} \mathrm{H}_{2} \mathrm{O}$, Aceton und Pentan gewaschen und $6 \mathrm{~h}$ i. Hochvak. getrocknet. Ausb. $141 \mathrm{mg}(96 \%)$; Zers.P. $245^{\circ} \mathrm{C}$. - IR (Nujol): $\tilde{v}=1958 \mathrm{~cm}^{-1}$ vs, $1934 \mathrm{~m}, 1744 \mathrm{~s} .-$ Wegen der geringen Löslichkeit von $\mathbf{4}$ in den gängigen Lösungsmitteln konnten keine NMR-Spektren gemessen werden. Gef. C 50.01, H 4.05 .

b) Dasselbe Produkt erhält man bei entsprechenden Umsetzungen aus einer roten $\mathrm{Na}\left[\mathrm{CpFe}(\mathrm{CO})_{2}\right]$-Lösung in $15 \mathrm{ml} \mathrm{THF}$, hergestellt aus $100 \mathrm{mg}(0.28 \mathrm{mmol})\left[\mathrm{Fe}(\mathrm{CO})_{2} \mathrm{Cp}\right]_{2}$ durch Reduktion mit Natriumamalgam, mit $199 \mathrm{mg}(45 \mathrm{mmol})\left[\mathrm{Cp}_{2} \mathrm{Fe}_{2}(\mathrm{CO})_{2}(\mu-\mathrm{CO})(\mu-\right.$ $\left.\left.\eta^{1}: \eta^{2}-\mathrm{CH}=\mathrm{CH}_{2}\right)\right] \mathrm{BF}_{4}$ oder aus einer Lösung von $100 \mathrm{mg}(0.28$ $\mathrm{mmol}) \mathrm{Na}\left[\mathrm{CpW}(\mathrm{CO})_{3}\right]$ in $15 \mathrm{ml} \mathrm{THF} \mathrm{mit} 123 \mathrm{mg}(0.28 \mathrm{mmol})$ $\left[\mathrm{Cp}_{2} \mathrm{Fe}_{2}(\mathrm{CO})_{2}(\mu-\mathrm{CO})\left(\mu-\eta^{1}: \eta^{2}-\mathrm{CH}=\mathrm{CH}_{2}\right)\right] \mathrm{BF}_{4}$. Die Rohprodukte werden wie bei a) durch Waschen mit polaren und unpolaren Lösungsmitteln gereinigt. Die Ausbeuten sind ebenfalls praktisch quantitativ.

c) Eine Lösung von $100 \mathrm{mg}(0.23 \mathrm{mmol})\left[\mathrm{Cp}_{2} \mathrm{Fe}_{2}(\mathrm{CO})_{2}(\mu-\mathrm{CO})(\mu-\right.$ $\left.\eta^{1}: \eta^{2}-\mathrm{CH}=\mathrm{CH}_{2}\right) \mathrm{BF}_{4}$ in $20 \mathrm{ml}$ THF wird mit $0.5 \mathrm{ml}(2.2 \mathrm{mmol}$ Na) $\mathrm{Na} / \mathrm{Hg}$ (100 mg Natrium auf $1 \mathrm{ml}$ Quecksilber) versetzt und langsam $1 \mathrm{~h}$ gerührt. Die ziegelrote Suspension wird vom Natriumamalgam abpipettiert. Nach Abtrennen des Lösungsmittels durch Zentrifugieren wird zweimal mit je $10 \mathrm{ml} \mathrm{H}_{2} \mathrm{O}$, Aceton und Pentan gewaschen und $6 \mathrm{~h}$ i. Hochvak. getrocknet. Die Ausbeute ist wie oben nahezu quantitativ; $72 \mathrm{mg}(89 \%)$.

$C p^{*} R u\left(\mu-\eta^{\prime}: \eta^{2}: \eta^{3}-C_{8} H_{12}\right) \operatorname{Re}(C O)_{5}$ (4): Man legt $493 \mathrm{mg}(1.0$ mmol) $\left[\mathrm{Cp} * \mathrm{Ru}\left(\mu-\eta^{2}: \eta^{4}-\mathrm{C}_{8} \mathrm{H}_{12}\right)\right] \mathrm{SO}_{3} \mathrm{CF}_{3}$ in $10 \mathrm{ml}$ THF bei $-70^{\circ} \mathrm{C}$ vor und tropft eine auf $-10^{\circ} \mathrm{C}$ gekühlte Lösung von $\mathrm{Na}\left[\operatorname{Re}(\mathrm{CO})_{5}\right]$ $\mathrm{zu}$, die zuvor aus $466 \mathrm{mg} \mathrm{Re} \mathrm{R}_{2}(\mathrm{CO})_{10}$ durch Reduktion mit Natriumamalgam in $10 \mathrm{ml}$ THF gewonnen wurde. Nach 3 min erhält man so eine klare, gelbe Lösung, die auf $0^{\circ} \mathrm{C}$ aufgetaut wird. Nach Entfernen des Lösungsmittels i. Hochvak. löst man 4 mit dreimal 15 $\mathrm{ml}$ Pentan aus dem orangefarbenen Rohprodukt. Durch Einengen auf $4 \mathrm{ml}$ und Abkühlen auf $-70^{\circ} \mathrm{C}$ fällt 4 als gelbes Pulver aus. Dieses wird aus Pentan umkristallisiert und $5 \mathrm{~h}$ i. Hochvak. getrocknet. Ausb. $617 \mathrm{mg}(92 \%)$; Schmp. $114-116^{\circ} \mathrm{C}$. - IR (Pentan): $\tilde{v}=2119 \mathrm{~cm}^{-1} \mathrm{~s}, 2043 \mathrm{w}, 2006 \mathrm{vs}, 1981 \mathrm{vs}, 1939 \mathrm{w} .{ }^{1} \mathrm{H}-\mathrm{NMR}$ $\left(400 \mathrm{MHz}, \mathrm{CD}_{2} \mathrm{Cl}_{2}\right): \delta=-0.05(\mathrm{~m}, 1 \mathrm{H}, 5-\mathrm{H}), 0.99(\mathrm{~d}, 1 \mathrm{H}, 8-\mathrm{H}$, $\left.{ }^{3} J_{7,8}=10.74 \mathrm{~Hz}\right), 1.55\left(\mathrm{~m}, 1 \mathrm{H}, 5^{\prime}-\mathrm{H}\right), 1.60\left(\mathrm{~s}, 15 \mathrm{H}, \mathrm{Cp}^{*}\right), 1.77(\mathrm{dd}$, $\left.1 \mathrm{H}, 1^{\prime}-\mathrm{H},{ }^{3} J_{1.2}=12.2,{ }^{2} \mathrm{~J}=11.23 \mathrm{~Hz}\right), 2.00(\mathrm{~m}, 2 \mathrm{H}, 6-\mathrm{H}), 2.12$ $\left(\mathrm{m}, 2 \mathrm{H}, 1,8^{\prime}-\mathrm{H},{ }^{3} J_{7.8}=8.3 \mathrm{~Hz}\right), 2.83(\mathrm{~m}, 1 \mathrm{H}, 2-\mathrm{H}), 3.02(\mathrm{dd}, 1 \mathrm{H}$, $\left.3-\mathrm{H},{ }^{3} J_{2.3}=6.83,{ }^{3} J_{2.3}=9.3 \mathrm{~Hz}\right), 3.12(\mathrm{~m}, 1 \mathrm{H}, 7-\mathrm{H}), 3.44(\mathrm{~m}, 1 \mathrm{H}$, 4-H). $-{ }^{13} \mathrm{C}-\mathrm{NMR}\left(100 \mathrm{MHz}, \mathrm{CD}_{2} \mathrm{Cl}_{2}\right): \delta=-1.78(\mathrm{C}-1), 9.04(\mathrm{Cp}-$ $\mathrm{CH}_{3}$ ), 27.27 (C-5), 38.5 (C-6), 45.44 (C-8), 68.95 (C-7), 70.56 (C4), 77.07 (C-2), 81.08 (C-3), $91.47\left(\mathrm{Cp}^{*}\right), 182.35\left(\mathrm{ReCO}_{\mathrm{ax}}\right), 187.35$ $\left(\mathrm{ReCO}_{\mathrm{äq}}\right)$. $-\mathrm{C}_{23} \mathrm{H}_{27} \mathrm{O}_{5} \mathrm{ReRu}(670.7)$ : ber. $\mathrm{C} 41.19, \mathrm{H} 4.06$; gef. C 41.25, H 4.08 .

$C p^{*} R u\left(\mu-\eta^{1}: \eta^{2}: \eta^{3}-C_{8} H_{12}\right) \mathrm{Fe}(\mathrm{CO})_{2} C p(5): 265 \mathrm{mg}(0.75 \mathrm{mmol})$ $\left[\mathrm{CpFe}(\mathrm{CO})_{2}\right]_{2}$ werden in $10 \mathrm{ml}$ THF mit Natriumamalgam bei Raumtemp. reduziert. Die rote Lösung wird auf $-20^{\circ} \mathrm{C}$ vorgekühlt und bei $-70^{\circ} \mathrm{C}$ langsam zu einer Suspension von $617 \mathrm{mg}(1.25$ $\mathrm{mmol})\left[\mathrm{Cp} * \mathrm{Ru}\left(\mu-\eta^{2}: \eta^{4}-\mathrm{C}_{8} \mathrm{H}_{12}\right)\right] \mathrm{SO}_{3} \mathrm{CF}_{3}$ in $8 \mathrm{ml}$ THF getropft. Die dabei entstehende rote Lösung wird nach 5 min auf $-30^{\circ} \mathrm{C}$ erwärmt und vom Lösungsmittel befreit. Bei $-10^{\circ} \mathrm{C}$ extrahiert man den roten Rückstand zweimal mit je $15 \mathrm{ml}$ Pentan. Die Pentanlösung wird auf $5 \mathrm{ml}$ eingeengt und bei $-70^{\circ} \mathrm{C} 10 \mathrm{~min}$ gerührt. Der rote Pentanüberstand wird vom orangefarbenen Feststoff abgehoben und verworfen. Anschließend wäscht man 5 zweimal mit je 3 $\mathrm{ml}$ Pentan $\left(-70^{\circ} \mathrm{C}\right)$. Das orangefarbene Pulver wird $5 \mathrm{~h}$ i. Hochvak. getrocknet. Ausb. $514 \mathrm{mg}(79 \%)$; Zers.-P. $95^{\circ} \mathrm{C}$. - IR (Pentan): $\tilde{v}=1993 \mathrm{~cm}^{-1} \mathrm{~s}, 1926 \mathrm{~s} . \quad-{ }^{1} \mathrm{H}-\mathrm{NMR}\left(400 \mathrm{MHz}, \mathrm{CD}_{2} \mathrm{Cl}_{2}\right): \delta=$ $0.03(\mathrm{~m}, 1 \mathrm{H}, 5-\mathrm{H}), 1.01\left(\mathrm{~d}, 1 \mathrm{H}, 8-\mathrm{H},{ }^{3} J_{7.8}=11.2 \mathrm{~Hz}\right), 1.60(\mathrm{~s}, \mathrm{Cp}-$ $\left.\mathrm{CH}_{3}\right), 1.65\left(\mathrm{~m}, 1 \mathrm{H}, 5^{\prime}-\mathrm{H}\right), 2.05\left(\mathrm{~m}, 3 \mathrm{H}, 1^{\prime}, 6-\mathrm{H}\right), 2.16\left(\mathrm{~d}, 1 \mathrm{H}, 8^{\prime}-\right.$ $\left.\mathrm{H},{ }^{3} J_{7.8^{\prime}}=9.3 \mathrm{~Hz}\right), 2.37(\mathrm{~m}, 1 \mathrm{H}, 1-\mathrm{H}), 2.80\left(\mathrm{dd}, 1 \mathrm{H}, 3-\mathrm{H},{ }^{3} J_{2.3}=\right.$ $\left.3.9,{ }^{3} J_{3,4}=7.8 \mathrm{~Hz}\right), 3.16(\mathrm{~m}, 2 \mathrm{H}, 2,7-\mathrm{H}), 3.51(\mathrm{~m}, 1 \mathrm{H}, 4-\mathrm{H}), 4.76$ (s, Cp). $-{ }^{13} \mathrm{C}-\mathrm{NMR}\left(100 \mathrm{MHz}, \mathrm{CD}_{2} \mathrm{Cl}_{2}\right): \delta=9.3\left(\mathrm{Cp}-\mathrm{CH}_{3}\right), 10.4$ (C-1), 27.85 (C-5), 38.55 (C-6), 45.52 (C-8), 69.35 (C-7), 71.36 (C4), 74.5 (C-2), 82.07 (C-3), 86.13 (Cp), $91.51\left(\mathrm{Cp}^{*}\right), 218.5(\mathrm{FeCO})$. $-\mathrm{C}_{25} \mathrm{H}_{32} \mathrm{FeO}_{2} \mathrm{Ru}$ (521.4): ber. C 57.59, H 6.19; gef. C $57.77, \mathrm{H}$ 6.25 .

$\left[C p^{*} R u\left(\mu-\eta^{l}: \eta^{2}: \eta^{3}-C_{8} H_{12}\right)\right]_{2} O s(C O)_{4}(6):$ Man legt $150 \mathrm{mg}$ $(0.425 \mathrm{mmol}) \mathrm{Na}_{2}\left[\mathrm{Os}(\mathrm{CO})_{4}\right]$ in $8 \mathrm{ml} \mathrm{THF}$ bei $-70^{\circ} \mathrm{C}$ vor und tropft eine auf $-20^{\circ} \mathrm{C}$ gekühlte Lösung von $419 \mathrm{mg}(0.85 \mathrm{mmol})$ [ $\mathrm{Cp}^{*}$

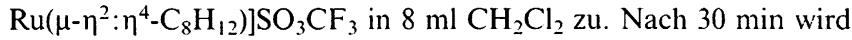
das Reaktionsgemisch langsam auf $0^{\circ} \mathrm{C}$ gebracht. Man engt die gelbe Lösung vollständig ein und extrahiert den Rückstand zweimal mit je $15 \mathrm{ml} \mathrm{CH}_{2} \mathrm{Cl}_{2} /$ Pentan (1:2). Die gesammelten Extrakte werden auf $4 \mathrm{ml}$ eingeengt und $30 \mathrm{~min}$ bei $-70^{\circ} \mathrm{C}$ gerührt. Das ausgefallene zitronengelbe Pulver wäscht man anschließend mit 5 ml Pentan $\left(-70^{\circ} \mathrm{C}\right) .6$ wird $10 \mathrm{~min}$ i. Hochvak. getrocknet, in $5 \mathrm{ml}$ $\mathrm{CH}_{2} \mathrm{Cl}_{2}$ gelöst und die Lösung mit $15 \mathrm{ml}$ Pentan überschichtet. Nach $2 \mathrm{~d}$ bei $-25^{\circ} \mathrm{C}$ erhält man 6 in Form von gelben plättchenförmigen Kristallen. Ausb. $248 \mathrm{mg}$ (59\%); Schmp. $128-131^{\circ} \mathrm{C}$. - IR (Nujol): $\tilde{v}=2106 \mathrm{~cm}^{-1} \mathrm{~m}, 2033 \mathrm{sh}, 2029 \mathrm{~m}, 2014$ vs. $-{ }^{1} \mathrm{H}-\mathrm{NMR}$ $\left(400 \mathrm{MHz}, \mathrm{CDCl}_{3}\right): \delta=-0.07(\mathrm{~m}, 1 \mathrm{H}, 5-\mathrm{H}), 1.01(\mathrm{~d}, 1 \mathrm{H}, 8-\mathrm{H}$, $\left.{ }^{3} J_{7,8}=10.75 \mathrm{~Hz}\right), 1.59\left(\mathrm{~s}, \mathrm{Cp}-\mathrm{CH}_{3}\right), 1.70\left(\mathrm{~m}, 2 \mathrm{H}, 1^{\prime}, 5^{\prime}-\mathrm{H}\right), 1.99$ $(\mathrm{m}, 2 \mathrm{H}, 6-\mathrm{H}), 2.12\left(\mathrm{~d}, 1 \mathrm{H}, 8^{\prime}-\mathrm{H},{ }^{3} J_{7,8^{\prime}}=8.8\right), 2.18(\mathrm{dd}, 1 \mathrm{H}, 1-\mathrm{H}$, $\left.{ }^{3} J_{1,2}=11.23,{ }^{2} J_{1,1^{\prime}}=3.42 \mathrm{~Hz}\right), 2.67(\mathrm{~m}, 1 \mathrm{H}, 2-\mathrm{H}), 2.94(\mathrm{~m}, 1 \mathrm{H}$, 3-H), $3.11(\mathrm{~m}, 1 \mathrm{H}, 7-\mathrm{H}), 3.44(\mathrm{~m}, 1 \mathrm{H}, 4-\mathrm{H}) .-{ }^{13} \mathrm{C}-\mathrm{NMR}(100$ $\left.\mathrm{MHz}, \mathrm{CDCl}_{3}\right): \delta=3.35(\mathrm{C}-1), 9.09\left(\mathrm{Cp}-\mathrm{CH}_{3}\right), 27.71$ (C-5), 38.32 (C-6), 45.51 (C-8), 69.24 (C-7), 71.08 (C-4), 74.3 (C-2), 81.25 (C3), $170.63,181.38(\mathrm{OsCO}) .-\mathrm{C}_{40} \mathrm{H}_{54} \mathrm{O}_{4} \mathrm{OsRu}_{2}$ (991.2): ber. C 48.47, H 5.49; gef. C 48.52, H 5.51 .

$$
\left[C p^{*} R u\left(\mu-\eta^{2}: \eta^{3}-C_{s} H_{12}-\right)\right]_{2} \text { (7) }
$$

a) Die durch Reduktion von $293 \mathrm{mg}(0.75 \mathrm{mmol}) \mathrm{Mn}_{2}(\mathrm{CO})_{10}$ in $12 \mathrm{ml}$ THF mit Natriumamalgam erhaltene, gelbgrüne Lösung tropft man bei $-70^{\circ} \mathrm{C}$ mittels einer Teflonkanüle zu einer Suspension von $617 \mathrm{mg}(1.25 \mathrm{mmol})\left[\mathrm{Cp} * \mathrm{Ru}\left(\mu-\eta^{2}: \eta^{4}-\mathrm{C}_{8} \mathrm{H}_{12}\right)\right] \mathrm{SO}_{3} \mathrm{CF}_{3}$ in $5 \mathrm{ml}$ THF. Es bildet sich sofort eine klare, orangefarbene Lösung, die bei $-10^{\circ} \mathrm{C}$ vom Lösungsmittel befreit wird. Man extrahiert 7 zusammen mit $\mathrm{Mn}_{2}(\mathrm{CO})_{10}$ mit dreimal $10 \mathrm{ml}$ Pentan aus dem Rückstand heraus. $\mathrm{Mn}_{2}(\mathrm{CO})_{10}$ wird durch Absublimieren bei $30^{\circ} \mathrm{C}$ i. Hochvak. $(8 \mathrm{~h})$ entfernt. Ausb. $202 \mathrm{mg}(47 \%)$; Zers.-P. $88^{\circ} \mathrm{C}$. ${ }^{1} \mathrm{H}-\mathrm{NMR}\left(400 \mathrm{MHz}, \mathrm{CDCl}_{3}\right): \delta=-0.09(\mathrm{~m}, 1 \mathrm{H}, 5-\mathrm{H}), 0.98(\mathrm{~d}$, $\left.1 \mathrm{H}, 8-\mathrm{H},{ }^{3} J_{7,8}=11.23 \mathrm{~Hz}\right), 1.57\left(\mathrm{~s}, \mathrm{Cp}-\mathrm{CH}_{3}\right), 1.66\left(\mathrm{~m}, 1 \mathrm{H}, 5^{\prime}-\mathrm{H}\right)$, $1.82(\mathrm{~m}, 2 \mathrm{H}, 6-\mathrm{H}), 2.02(\mathrm{~m}, 3 \mathrm{H}, 1,2-\mathrm{H}), 2.12\left(\mathrm{~m}, 1 \mathrm{H}, 8^{\prime}-\mathrm{H},{ }^{3} J_{8^{\prime} .7}=\right.$ $9.27 \mathrm{~Hz}), 2.88\left(\mathrm{dd}, 1 \mathrm{H}, 3-\mathrm{H},{ }^{3} J_{2.3}=6.84,{ }^{3} J_{3,4}=9.28 \mathrm{~Hz}\right), 3.18$ $(\mathrm{m}, 1 \mathrm{H}, 7-\mathrm{H}), 3.49(\mathrm{~m}, 1 \mathrm{H}, 4-\mathrm{H}) .-{ }^{13} \mathrm{C}-\mathrm{NMR}\left(100 \mathrm{MHz}, \mathrm{CDCl}_{3}\right)$ : $\delta=9.06\left(\mathrm{Cp}^{-\mathrm{CH}_{3}}\right), 18.45(\mathrm{C}-1), 27.85(\mathrm{C}-5), 38.24(\mathrm{C}-6), 45.21(\mathrm{C}-$ 8), 53.57 (C-4), 69.67 (C-7), 72.03 (C-2), 83.89 (C-3), 91.38 (Cp*). $-\mathrm{C}_{36} \mathrm{H}_{54} \mathrm{Ru}_{2}$ (689.0): ber. C 62.76, H 7.90; gef. C 61.65, H 7.82.

b) $500 \mathrm{mg}(1.02 \mathrm{mmol})\left[\mathrm{Cp} * \mathrm{Ru}\left(\mu-\eta^{2}: \eta^{4}-\mathrm{C}_{8} \mathrm{H}_{12}\right)\right] \mathrm{SO}_{3} \mathrm{CF}_{3}$ werden in $20 \mathrm{ml}$ THF suspendiert, auf $-20^{\circ} \mathrm{C}$ vorgekühlt und mit $1 \mathrm{ml}$ 0.9 proz. Natriumamalgam versetzt. Man rührt $30 \mathrm{~min}$ bei $-20^{\circ} \mathrm{C}$ und weitere $2 \mathrm{~h}$ bei Raumtemp. Die orangefarbene Lösung wird vom Lösungsmittel befreit und wie oben beschrieben mit Pentan behandelt. Nach Trocknen i. Hochvak. erhält man ein orangefarbenes Pulver. Ausb. $234 \mathrm{mg}(68 \%)$; Zers.-P. $88^{\circ} \mathrm{C}$. $-\mathrm{C}_{36} \mathrm{H}_{54} \mathrm{Ru}_{2}$ (689.0): ber. C 62.76, H 7.90; gef. C 62.77, H 7.81. 
$\left[\left(C p^{*} R u\left(\mu-\eta^{2}: \eta^{3}-C_{8} H_{12}\right)\right]\left[C p M o(C O)_{3}\right]\right.$ : Eine Suspension von $283 \mathrm{mg}(0.57 \mathrm{mmol})\left[\left(\mathrm{Cp} * \mathrm{Ru}\left(\mu-\eta^{2}: \eta^{4}-\mathrm{C}_{8} \mathrm{H}_{12}\right)\right] \mathrm{SO}_{3} \mathrm{CF}_{3}\right.$ in $5 \mathrm{ml} \mathrm{THF}$ wird bei $-70^{\circ} \mathrm{C}$ mit einer Lösung von $\mathrm{Na}\left[\mathrm{CpMo}(\mathrm{CO})_{3}\right]$, die durch $\mathrm{Na} / \mathrm{Hg}$-Reduktion aus $150 \mathrm{mg}\left[\mathrm{CpMo}(\mathrm{CO})_{3}\right]_{2}$ in $10 \mathrm{ml} \mathrm{THF} \mathrm{er-}$ zeugt wurde, versetzt. Die gelbe Reaktionslösung wird langsam auf Raumtemp. erwärmt, weitere $20 \mathrm{~min}$ gerührt und schließlich i. Hochvak. vom Lösungsmittel befreit. Die Extraktion mit dreimal $5 \mathrm{ml} \mathrm{CH} \mathrm{Cl}_{2}$ liefert eine gelbe Lösung, die auf $3 \mathrm{ml}$ eingeengt und bei $-20^{\circ} \mathrm{C}$ mit $20 \mathrm{ml}$ Ether versetzt wird. Der zitronengelbe Niederschlag wird erneut aus $\mathrm{CH}_{2} \mathrm{Cl}_{2} /$ Ether umgefällt und i.Vak. getrocknet. Ausb. $292 \mathrm{mg}(87 \%)$; Zers. $\mathrm{P}$. $130^{\circ} \mathrm{C}$. $-\mathrm{C}_{26} \mathrm{H}_{32} \mathrm{MoO}_{3} \mathrm{Ru}$ (589.6): ber. C 52.97, H 5.47; gef. C 51.68, H 5.37

(OC) $\mathrm{Fe}\left(\eta^{4}-\mathrm{C}_{6} H_{10}\right)\left(\mu-\eta^{\prime}: \eta^{4}-\mathrm{C}_{7} \mathrm{H}_{9}\right) \operatorname{Re}(\mathrm{CO})_{5}(\mathbf{8})$ : Man suspendiert $207 \mathrm{mg}(0.6 \mathrm{mmol})\left[(\mathrm{OC}) \mathrm{Fe}\left(\eta^{4}-\mathrm{C}_{6} \mathrm{H}_{10}\right)\left(\eta^{5}-\mathrm{C}_{7} \mathrm{H}_{9}\right)\right] \mathrm{BF} \mathrm{B}_{4}$ in $5 \mathrm{ml}$ THF und tropft bei $-78^{\circ} \mathrm{C}$ unter Rühren eine orangefarbene Lösung von $\mathrm{NaRe}(\mathrm{CO})_{5}$ [dargestellt aus $280 \mathrm{mg}$ (0.43 mmol) $\left.\operatorname{Re}_{2}(\mathrm{CO})_{10}\right]$ zu. Nach $10 \mathrm{~min}$. Rühren erwärmt man das Reaktionsgemisch auf $-10^{\circ} \mathrm{C}$, entfernt das Lösungsmittel i. Hochvak. und extrahiert bei $0^{\circ} \mathrm{C}$ dreimal mit $15 \mathrm{ml}$ Pentan. Die vereinigten Extrakte werden auf $4 \mathrm{ml}$ eingeengt, und die überstehende Lösung wird vom festen, orangefarbenen 8 abgetrennt. Der zunächst anfallende wachsartige Feststoff kann durch Lösen in $8 \mathrm{ml}$ Pentan und langsames Abkühlen auf $-70^{\circ} \mathrm{C}$ in orangefarbene Kristalle übergeführt werden. Ausb. $203 \mathrm{mg}(58 \%)$; Zers.-P. $72^{\circ} \mathrm{C}$. - IR (Pentan): $\bar{v}=2120 \mathrm{~cm}^{-1} \mathrm{~s}, 2048 \mathrm{~m}, 2010 \mathrm{vs}, 1985 \mathrm{vs}, 1964 \mathrm{~s} .-{ }^{1} \mathrm{H}-\mathrm{NMR}$ $\left(400 \mathrm{MHz}, \mathrm{CD}_{2} \mathrm{Cl}_{2}\right): \delta=-0.52\left(\mathrm{~d}, 2 \mathrm{H}, \mathrm{CH}_{2, s y n},{ }^{2} J=8.3 \mathrm{~Hz}\right), 0.91$ (d, $\left.2 \mathrm{H}, \mathrm{CH}_{2 a n t i},{ }^{2} J=13.7 \mathrm{~Hz}\right), 1.69\left(\mathrm{~m}, 2 \mathrm{H}, 6^{\prime}, 7^{\prime}-\mathrm{H}\right), 1.75(\mathrm{~s}, 3 \mathrm{H}$, $\mathrm{Me}), 1.85(\mathrm{~s}, 3 \mathrm{H}, \mathrm{Me}), 1.94(\mathrm{~m}, 1 \mathrm{H}, 7-\mathrm{H}), 2.11(\mathrm{~m}, 1 \mathrm{H}, 6-\mathrm{H})$, $2.42(\mathrm{~m}, 1 \mathrm{H}, 1-\mathrm{H}), 2.79(\mathrm{dd}, 1 \mathrm{H}, 4-\mathrm{H}), 3.64(\mathrm{~m}, 1 \mathrm{H}, 5-\mathrm{H}), 3.78$ $(\mathrm{m}, 1 \mathrm{H}, 3-\mathrm{H}), 3.98(\mathrm{~m}, 1 \mathrm{H}, 2-\mathrm{H}) .{ }^{13} \mathrm{C}-\mathrm{NMR}(100 \mathrm{MHz}$, $\left.\mathrm{CD}_{2} \mathrm{Cl}_{2}\right): \delta=17.35,17.82(\mathrm{Me}) ; 23.29(\mathrm{C}-5) ; 35.94,41.12(\mathrm{C}-6,7)$; 41.67, 41.76 (C-8,11), 58.45 (C-1); 80.17 (C-4); 82.80, 89.21 (C-2,3); 90.76, 91.75 (C-9,10); 181.02, $186.62(\mathrm{ReCO}) ; 213.29$ (FeCO). $\mathrm{C}_{19} \mathrm{H}_{19} \mathrm{FeO}_{6} \mathrm{Re}(585.4)$ : ber. C 38.98, H 3.27; gef. C 39.08, H 3.27.

(OC) $\mathrm{Fe}\left(\eta^{4}-\mathrm{C}_{6} \mathrm{H}_{8}\right)\left(\mu-\eta^{\prime}: \eta^{4}-\mathrm{C}_{7} \mathrm{H}_{9}\right) \operatorname{Re}(\mathrm{CO})_{5}$ (9): Eine Lösung von $\mathrm{Na}\left[\mathrm{Re}(\mathrm{CO})_{5}\right]$, die durch Reduktion von $340 \mathrm{mg}(0.52 \mathrm{mmol})$ $\operatorname{Re}_{2}(\mathrm{CO})_{10}$ mit Natriumamalgam in $10 \mathrm{ml}$ THF erhalten wurde, kühlt man auf $-20^{\circ} \mathrm{C}$. Mit einer Teflonkanüle tropft man bei $-70^{\circ} \mathrm{C}$ die Lösung zu einer Suspension von $258 \mathrm{mg}(0.75 \mathrm{mmol})$ $\left[(\mathrm{OC}) \mathrm{Fe}\left(\eta^{4}-\mathrm{C}_{6} \mathrm{H}_{8}\right)\left(\eta^{5}-\mathrm{C}_{7} \mathrm{H}_{9}\right)\right] \mathrm{BF}_{4}$ in $8 \mathrm{ml}$ THF. Es bildet sich sofort eine klare, orangefarbene Lösung, die auf $-20^{\circ} \mathrm{C}$ aufgetaut wird. Nach Entfernen des Lösungsmittels bei dieser Temp. extrahiert man bei $0^{\circ} \mathrm{C}$ dreimal mit $15 \mathrm{ml}$ Pentan. Die vereinigten Extrakte werden auf $4 \mathrm{ml}$ eingeengt, und die überstehende Lösung wird vom orangefarbenen, feinkristallinen 9 abgetrennt. Durch erneutes Auflösen in $10 \mathrm{ml}$ Pentan bei Raumtemp. und anschließendes Kühlen auf $-25^{\circ} \mathrm{C}$ über Nacht wird 9 in Form von orangefarbenen Kristallen erhalten. Ausb. $346 \mathrm{mg}(79 \%)$; Zers.-P. $80^{\circ} \mathrm{C}$. - IR (Pentan): $\tilde{v}=2120 \mathrm{~cm}^{-1} \mathrm{w}, 2049 \mathrm{~m}, 2011 \mathrm{vs}, 1985 \mathrm{vs}, 1958 \mathrm{~m} .-{ }^{1} \mathrm{H}-\mathrm{NMR}$ $\left(400 \mathrm{MHz}, \mathrm{CD}_{2} \mathrm{Cl}_{2}\right): \delta=1.08(\mathrm{~m}, 2 \mathrm{H}, 12-\mathrm{H}), 1.69(\mathrm{~m}, 2 \mathrm{H}, 7-\mathrm{H})$, $1.90\left(\mathrm{~m}, 3 \mathrm{H}, 6^{\prime}, 13-\mathrm{H}\right), 2.10(\mathrm{~m}, 1 \mathrm{H}, 6-\mathrm{H}), 2.41(\mathrm{~m}, 1 \mathrm{H}, 8-\mathrm{H}), 2.49$ $(\mathrm{m}, 1 \mathrm{H}, 11-\mathrm{H}), 2.62(\mathrm{~m}, 1 \mathrm{H}, 1-\mathrm{H}), 2.77\left(\mathrm{dd}, 1 \mathrm{H}, 4-\mathrm{H},{ }^{3} J_{3,4}=12.7\right.$, $\left.{ }^{3} J_{4,5}=3.4 \mathrm{~Hz}\right), 3.81(\mathrm{~m}, 1 \mathrm{H}, 5-\mathrm{H}), 4.28(\mathrm{~m}, 1 \mathrm{H}, 3-\mathrm{H}), 4.38(\mathrm{~m}$, $1 \mathrm{H}, 9-\mathrm{H}), 4.50(\mathrm{~m}, 1 \mathrm{H}, 2-\mathrm{H}), 4.58(\mathrm{~m}, 1 \mathrm{H}, 10-\mathrm{H}) .-{ }^{13} \mathrm{C}-\mathrm{NMR}$ $\left(100 \mathrm{MHz}, \mathrm{CD}_{2} \mathrm{Cl}_{2}\right): \delta=23.26(\mathrm{C}-5), 24.37,24.59(\mathrm{C}-12,13), 36.04$, 41.93 (C-6,7), 59.43 (C-1), 60.47, 61.22 (C-8,11), 77.86 (C-4), 81.14, 84.36 (C-9,10), 82.1, $84.36(\mathrm{C}-2,3), 181.47\left(\mathrm{ReCO}_{\mathrm{ax}}\right), 186.99$ (Re$\mathrm{CO}_{\text {ăq }}$ ). $-\mathrm{C}_{19} \mathrm{H}_{17} \mathrm{FeO}_{6} \mathrm{Re}(583.4)$ : ber. C 39.12, H 2.94; gef. C 39.42, H 2.96.

[(OC) $\left.\mathrm{Fe}\left(\eta^{4}-\mathrm{C}_{6} \mathrm{H}_{8}\right)\left(\mu-\eta^{\prime}: \eta^{4}-\mathrm{C}_{7} \mathrm{H}_{9}\right)\right]_{2} \mathrm{Os}(\mathrm{CO})_{4}$ (10): Man löst $309 \mathrm{mg}(0.9 \mathrm{mmol})\left[(\mathrm{OC}) \mathrm{Fe}\left(\eta^{4}-\mathrm{C}_{6} \mathrm{H}_{8}\right)\left(\eta^{5}-\mathrm{C}_{7} \mathrm{H}_{9}\right)\right] \mathrm{BF}_{4}$ in $15 \mathrm{ml}$ $\mathrm{CH}_{2} \mathrm{Cl}_{2}$ und gibt bei $-70^{\circ} \mathrm{C} 157 \mathrm{mg}(0.45 \mathrm{mmol}) \mathrm{Na}_{2}\left[\mathrm{Os}(\mathrm{CO})_{4}\right] \mathrm{zu}$.
Nach $1 \mathrm{~h}$ erhält man eine gelbe Lösung, die bei $-10^{\circ} \mathrm{C}$ auf $5 \mathrm{ml}$ eingeengt wird. Nach Zugabe von $20 \mathrm{ml}$ Pentan rührt man $30 \mathrm{~min}$ bei $-70^{\circ} \mathrm{C}$. Anschließend wird die entstandene Suspension zentrifugiert. Die überstehende Lösung wird abgetrennt, auf $3 \mathrm{ml}$ eingeengt und auf $-70^{\circ} \mathrm{C}$ abgekühlt. Das ausgefallene, orangefarbene Pulver wird noch zweimal bei $-70^{\circ} \mathrm{C}$ mit je $3 \mathrm{ml}$ Pentan gewaschen und i. Hochvak. getrocknet. Ausb. $132 \mathrm{mg}(36 \%)$; Zers.-P. $61^{\circ} \mathrm{C}$. IR (Pentan): $\bar{v}=2116 \mathrm{~cm}^{-1} \mathrm{~m}, 2038 \mathrm{vs}, 2033 \mathrm{vs}, 2004 \mathrm{~s}, 1956 \mathrm{~s}$. ${ }^{1} \mathrm{H}-\mathrm{NMR}\left(400 \mathrm{MHz}, \mathrm{CD}_{2} \mathrm{Cl}_{2}\right): \delta=1.13(\mathrm{~m}, 2 \mathrm{H}, 12-\mathrm{H}), 1.92(\mathrm{~m}$, $6 \mathrm{H}, 6,7,13-\mathrm{H}), 2.52(\mathrm{~m}, 4 \mathrm{H}, 1,4,8,11-\mathrm{H}), 3.83(\mathrm{~m}, 1 \mathrm{H}, 5-\mathrm{H}), 4.48$ (m, 4H, 2,3,9,10-H). ${ }^{13} \mathrm{C}-\mathrm{NMR}\left(100 \mathrm{MHz}, \mathrm{CD}_{2} \mathrm{Cl}_{2}\right): \delta=24.04$, 24.37 (C-12,13), 24.64 (C-5), 32.3, 35.72 (C-6,7), 59.63 (C-1), 62.0, 62.11 (C-8,11), 79.31 (C-4), 82.38, 82.54 (C-9,10), 82.14, 84.87 (C2,3), 179.85 (OsCO). $-\mathrm{C}_{32} \mathrm{H}_{34} \mathrm{Fe}_{2} \mathrm{O}_{6} \mathrm{Os}$ (816.5): ber. $\mathrm{C} 47.07, \mathrm{H}$ 4.20; gef. C 47.85, H 4.72 .

[(OC) Fe $\left.\left(\eta^{4}-C_{6} H_{8}\right)\left(\mu-\eta^{l}: \eta^{4}-C_{7} H_{9}-\right)\right]_{2}(\mathbf{1 1}): 859 \mathrm{mg}(2.5 \mathrm{mmol})$ $\left[(\mathrm{OC}) \mathrm{Fe}\left(\eta^{4}-\mathrm{C}_{6} \mathrm{H}_{8}\right)\left(\eta^{5}-\mathrm{C}_{7} \mathrm{H}_{9}\right)\right] \mathrm{BF}_{4}$ werden in $15 \mathrm{ml}$ THF suspendiert. Die Reduktion mit Natriumamalgam erfolgt bei $-15^{\circ} \mathrm{C}$ durch 2stdg. Rühren. Dabei kommt es zu einer Klärung der Suspension, und man erhält schließlich eine tiefrote Lösung. Diese wird abgetrennt und bei $0^{\circ} \mathrm{C}$ vom Lösungsmittel befreit. Aus dem klebrigen Rückstand löst man 11 durch dreimalige Extraktion mit je $10 \mathrm{ml}$ Pentan heraus. Die gesammelten Extrakte werden bis auf $3 \mathrm{ml}$ reduziert. Kühlen auf $-70^{\circ} \mathrm{C}$ liefert eine ölige orangefarbene Substanz. Diese wird nach Verwerfen des Pentanüberstandes mit 5 $\mathrm{ml}$ Pentan versetzt und bei $-70^{\circ} \mathrm{C} 1 \mathrm{~h}$ kräftig gerührt. Man erhält schließlich ein orangefarbenes Pulver. Ausb. $308 \mathrm{mg}$ (48\%); Zers. P. $51{ }^{\circ} \mathrm{C}$. - IR (Pentan): $\tilde{v}=1963 \mathrm{~cm}^{-1}$ s. $-{ }^{\prime} \mathrm{H}-\mathrm{NMR}(400 \mathrm{MHz}$, $\left.\mathrm{CDCl}_{3}\right): \delta=1.04(\mathrm{~m}, 3 \mathrm{H}, 5,12-\mathrm{H}), 1.97(\mathrm{~m}, 6 \mathrm{H}, 6,7,13-\mathrm{H}), 2.58$ $(\mathrm{m}, 4 \mathrm{H}, 1,4,8,11-\mathrm{H}), 4.46(\mathrm{~m}, 4 \mathrm{H}, 2,3,9,10-\mathrm{H}) .-{ }^{13} \mathrm{C}-\mathrm{NMR}(100$ $\left.\mathrm{MHz}, \mathrm{CDCl}_{3}\right): \delta=23.91,24.16(\mathrm{C}-12,13), 28.76(\mathrm{C}-5), 32.07,32.57$ (C-6,7), 59.09, 59.29, 61.58, 61.74 (C-1,4,8,11), 80.91, 81.95, 83.13, $84.13(\mathrm{C}-2,3,9,10)$. $-\mathrm{C}_{28} \mathrm{H}_{34} \mathrm{Fe}_{2} \mathrm{O}_{2}$ (514.3): ber. C 65.40, H 6.66; gef. C $64.79, \mathrm{H} 6.54$.

$\mathrm{CpCo}\left(\mu-\eta^{\prime}: \eta^{4}-\mathrm{C}_{6} \mathrm{H}_{7}\right) \operatorname{Re}(\mathrm{CO})_{5}(\mathbf{1 2})$ : Aus $200 \mathrm{mg}(0.31 \mathrm{mmol})$ $\mathrm{Re}_{2}(\mathrm{CO})_{10}$ wird in $15 \mathrm{ml}$ THF durch Reduktion mit $\mathrm{Na} / \mathrm{Hg}$ eine gelborangefarbene $\mathrm{Na}\left[\operatorname{Re}(\mathrm{CO})_{5}\right]$-Lösung hergestellt. Diese wird auf $-70^{\circ} \mathrm{C}$ gekühlt und portionsweise mit $129 \mathrm{mg}(0.37 \mathrm{mmol})$ $\left[\mathrm{CpCo}\left(\eta^{5}-\mathrm{C}_{6} \mathrm{H}_{7}\right)\right] \mathrm{PF}_{6}$ versetzt. Es bildet sich rasch eine rotorangefarbene Lösung. Nach etwa $30 \mathrm{~min}$. Rühren wird langsam auf $-40^{\circ} \mathrm{C}$ erwärmt und anschließend das Lösungsmittel bei dieser Temp. i. Hochvak. entfernt. Das Rohprodukt wird zweimal mit je $5 \mathrm{ml}$ Pentan bei $-30^{\circ} \mathrm{C}$ gewaschen, um in geringen Mengen vorhandenes $\operatorname{Re}_{2}(\mathrm{CO})_{10} \mathrm{zu}$ entfernen. Durch dreimalige Extraktion mit je $15 \mathrm{ml}$ Pentan bei $-30^{\circ} \mathrm{C}$ und Entfernen des Lösungsmittels i. Vak. erhält man 12 als orangeroten Feststoff, der noch $6 \mathrm{~h}$ bei $-30^{\circ} \mathrm{C}$ i. Hochvak. getrocknet wird. Ausb. $82 \mathrm{mg}(42 \%)$; Zers.-P. $45^{\circ} \mathrm{C}$. - IR (Nujol): $\tilde{v}=2114 \mathrm{~cm}^{-1} \mathrm{w}, 2026 \mathrm{~m}, 2006 \mathrm{vs}, 1993 \mathrm{~s}$, 1980 m. - 'H-NMR (90 MHz, $\left.\mathrm{CD}_{2} \mathrm{Cl}_{2}\right): \delta=0.87-1.36(\mathrm{~m}, 2 \mathrm{H}$, $\left.1,2_{\text {exo }}-\mathrm{H}\right), 1.66-2.22\left(\mathrm{~m}, 2 \mathrm{H}, 2_{\text {cndo }}, 6-\mathrm{H}\right), 3.24(\mathrm{~m}, 1 \mathrm{H}, 3-\mathrm{H}), 4.27$ (m, 1 H, 5-H), $4.71(\mathrm{~s}, 5 \mathrm{H}, \mathrm{Cp}), 4.97(\mathrm{~m}, 1 \mathrm{H}, 4-\mathrm{H})$. - 12 zersetzt sich in Lösung zu schnell, um saubere ${ }^{13} \mathrm{C}-\mathrm{NMR}$-Spektren zu erhalten. $-\mathrm{C}_{16} \mathrm{H}_{11} \mathrm{CoO}_{5} \mathrm{Re}$ (529.8); ber. C 36.45, H 2.09; gef. $\mathrm{C}$ $35.54, \mathrm{H} 2.26$.

$C p C o\left(\mu-\eta^{\prime}: \eta^{4}-C_{7} H_{9}\right) \operatorname{Re}(C O)_{5}(13):$ Aus $200 \mathrm{mg}(0.31 \mathrm{mmol})$ $\operatorname{Re}_{2}(\mathrm{CO})_{10}$ wird in $15 \mathrm{ml}$ THF durch Reduktion mit Natriumamalgam eine gelborangefarbene $\mathrm{Na}\left[\operatorname{Re}(\mathrm{CO})_{5}\right]$-Lösung hergestellt. Diese wird auf $-70^{\circ} \mathrm{C}$ gekühlt und portionsweise mit $134 \mathrm{mg}(0.37$ mmol) $\left[\mathrm{CpCo}\left(\eta^{5}-\mathrm{C}_{7} \mathrm{H}_{9}\right)\right] \mathrm{PF}_{6}$ versetzt. Es bildet sich rasch eine rotorangefarbene Lösung. Nach etwa $30 \mathrm{~min}$. Rühren wird langsam auf $-40^{\circ} \mathrm{C}$ erwärmt und anschließend das Lösungsmittel bei dieser Temp. i. Hochvak. entfernt. Das Rohprodukt wird bei $-30^{\circ} \mathrm{C}$ ein- 
mal mit $5 \mathrm{ml}$ Pentan gewaschen, um in geringen Mengen vorhandenes $\operatorname{Re}_{2}(\mathrm{CO})_{10} \mathrm{zu}$ entfernen. Durch zweimalige Extraktion mit je $15 \mathrm{ml}$ Pentan bei $-30^{\circ} \mathrm{C}$ und Entfernen des Lösungsmittels i. Vak. erhält man 13 als rotvioletten Feststoff, der noch $6 \mathrm{~h}$ bei $-30^{\circ} \mathrm{C}$ i. Hochvak. getrocknet wird und sich bei Raumtemp. rasch zersetzt. Ausb. $133 \mathrm{mg}(66 \%)$; Zers.-P. $50^{\circ} \mathrm{C}$. - IR (Nujol): $\tilde{v}=2116 \mathrm{~cm}^{-1}$ m, $2041 \mathrm{~m}, 2008$ vs, $1981 \mathrm{~s}, 1960 \mathrm{~m}$. - ${ }^{1} \mathrm{H}-\mathrm{NMR}(90 \mathrm{MHz}$, $\left.\mathrm{CD}_{2} \mathrm{Cl}_{2}\right): \delta=1.09-2.25(\mathrm{~m}, 6 \mathrm{H}, 1,3,7-\mathrm{H}), 3.07(\mathrm{~m}, 1 \mathrm{H}, 4-\mathrm{H}), 4.23$ (m, 1 H, 6-H), 4.64 (s, 5H, Cp), $4.71(\mathrm{~m}, 1 \mathrm{H}, 5-\mathrm{H}) .-{ }^{13} \mathrm{C}-\mathrm{NMR}$ (22.5 MHz, $\mathrm{CD}_{2} \mathrm{Cl}_{2},-20^{\circ} \mathrm{C}$ ): $\delta=23.95(\mathrm{C}-1), 36.14$ (C-2), 40.89 (C-3), 46.80 (C-7), 68.39 (C-4), 74.83 (C-6), 80.91 (Cp), 81.27 (C5), $187.43(\mathrm{ReCO}) .-\mathrm{C}_{17} \mathrm{H}_{14} \mathrm{CoO}_{5} \mathrm{Re}$ (543.8): ber. $\mathrm{C} 37.54, \mathrm{H} 2.59$; gef. C 35.36, H 2.36 .

$\left[\mathrm{CpCo}\left(\eta^{4}-\mathrm{C}_{6} \mathrm{H}_{7}-\right)\right]_{2}(\mathbf{1 4}): \mathrm{Zu}$ einer gelbgrünen Lösung von $\mathrm{Na}\left[\mathrm{Mn}(\mathrm{CO})_{5}\right]$ in $15 \mathrm{ml} \mathrm{THF}$, dargestellt aus $100 \mathrm{mg}(0.26 \mathrm{mmol})$ $\mathrm{Mn}_{2}(\mathrm{CO})_{10}$ durch Reduktion mit Natriumamalgam, werden bei $-70^{\circ} \mathrm{C} 153 \mathrm{mg}(0.44 \mathrm{mmol})\left[\mathrm{CpCo}\left(\eta^{5}-\mathrm{C}_{6} \mathrm{H}_{7}\right)\right] \mathrm{PF}_{6}$ gegeben. Nach etwa $1 \mathrm{~h}$ hat sich eine gelbbraune Lösung gebildet. Man läßt auf Raumtemp. kommen, entfernt das Lösungsmittel i. Hochvak. und extrahiert viermal mit je $10 \mathrm{ml}$ Pentan. Das Lösungsmittel wird wieder i. Hochvak. entfernt und das im Rückstand enthaltene $\mathrm{Mn}_{2}(\mathrm{CO})_{10}$ durch Sublimation $(4 \mathrm{~h})$ bei etwa $50^{\circ} \mathrm{C}$ vollständig abgetrennt. Um 14 von geringen Mengen Zersetzungsprodukten abzutrennen, wird nochmals mit zweimal $15 \mathrm{ml}$ Pentan extrahiert, das Lösungsmittel entfernt und der orangefarbene Feststoff $6 \mathrm{~h}$ i. Hochvak. getrocknet. Ausb. $50 \mathrm{mg}(57 \%)$; Zers.-P. $155^{\circ} \mathrm{C}$. $-{ }^{1} \mathrm{H}$ $\operatorname{NMR}\left(90 \mathrm{MHz}, \mathrm{C}_{6} \mathrm{D}_{6}\right): \delta=0.25\left(\mathrm{~m}, 2 \mathrm{H}, 2,2^{\prime}{ }_{\text {exo }}-\mathrm{H}\right), 1.57(\mathrm{~m}, 4 \mathrm{H}$, $\left.1,1^{\prime}, 2,2^{\prime}{ }_{\text {endo }}-\mathrm{H}\right), 2.87\left(\mathrm{~m}, 4 \mathrm{H}, 3,3^{\prime}, 6,6^{\prime}-\mathrm{H}\right), 4.58(\mathrm{~s}, 10 \mathrm{H}, \mathrm{Cp}), 4.81$ $\left(\mathrm{m}, 4 \mathrm{H}, 4,4^{\prime}, 5,5^{\prime}-\mathrm{H}\right) .-{ }^{13} \mathrm{C}-\mathrm{NMR}\left(22.5 \mathrm{MHz}, \mathrm{C}_{6} \mathrm{D}_{6}\right): \delta=30.32$ (C-2), 47.59 (C-1), 49.71 (C-3), 56.62 (C-6), 77.19 (C-4,5), 79.69 (Cp). $-\mathrm{C}_{22} \mathrm{H}_{24} \mathrm{Co}_{2}$ (406.3): ber. C 65.03, H 5.95; gef. C 63.85, H 6.10 .

$\left[\mathrm{CpCo}\left(\eta^{4}-\mathrm{C}_{7} \mathrm{H}_{9}\right)-\right]_{2}(\mathbf{1 5})$ : $\mathrm{Zu}$ einer gelbgrünen Lösung von $\mathrm{Na}\left[\mathrm{Mn}(\mathrm{CO})_{5}\right]$ in $15 \mathrm{ml} \mathrm{THF}$, dargestellt aus $100 \mathrm{mg}(0.26 \mathrm{mmol})$ $\mathrm{Mn}_{2}(\mathrm{CO})_{10}$ durch Reduktion mit Natriumamalgam, werden bei $-70^{\circ} \mathrm{C} 159 \mathrm{mg}(0.44 \mathrm{mmol})\left[\mathrm{CpCo}\left(\eta^{5}-\mathrm{C}_{7} \mathrm{H}_{9}\right)\right] \mathrm{PF}_{6}$ gegeben. Nach etwa $1 \mathrm{~h}$ hat sich eine gelbbraune Lösung gebildet. Man läßt auf Raumtemp. kommen und entfernt das Lösungsmittel i. Hochvak. Die weitere Reinigung erfolgt wie bei 14. Man erhält so den Komplex 15 als rotorangefarbenen Feststoff. Ausb. $67 \mathrm{mg} \mathrm{(70 \% );} \mathrm{Zers.}$ ab $144^{\circ} \mathrm{C} .-{ }^{1} \mathrm{H}-\mathrm{NMR}\left(90 \mathrm{MHz}, \mathrm{C}_{6} \mathrm{D}_{6}\right): \delta=1.06-1.48(\mathrm{~m}, 4 \mathrm{H}$, $\left.1,1^{\prime}, 2,2^{\prime}{ }_{\text {exo }}-\mathrm{H}\right), 1.60-1.85\left(\mathrm{~m}, 6 \mathrm{H}, 2,2^{\prime}{ }_{\text {endo }}, 3,3^{\prime}{ }_{\text {exo und endo }}-\mathrm{H}\right), 2.70$ $\left(\mathrm{m}, 2 \mathrm{H}, 7,7^{\prime}-\mathrm{H}\right), 3.01\left(\mathrm{~m}, 2 \mathrm{H}, 4,4^{\prime}-\mathrm{H}\right), 4.57(\mathrm{~s}, 10 \mathrm{H}, \mathrm{Cp})$, $4.63-4.93\left(\mathrm{~m}, 4 \mathrm{H}, 5,5^{\prime}, 6,6^{\prime}-\mathrm{H}\right) .-{ }^{13} \mathrm{C}-\mathrm{NMR}\left(22.5 \mathrm{MHz}, \mathrm{C}_{6} \mathrm{D}_{6}\right)$ : $\delta=24.33$ (C-2), 31.46 (C-3), 47.97 (C-1), 49.18 (C-7), 50.89 (C-4), $80.38(\mathrm{C}-5,6), 81.29(\mathrm{Cp}) .-\mathrm{C}_{24} \mathrm{H}_{28} \mathrm{Co}_{2}$ (434.3): ber. $\mathrm{C} 66.36, \mathrm{H}$ 6.50 ; gef. C $65.35, \mathrm{H} 6.41$.

Herrn Professor Ekkehard Lindner zum 60. Geburtstag gewidmet.

[1] XXIX. Mitteilung: S. Hüffer, M. Wieser, K. Polborn, W. Beck, J. Organomet. Chem., im Druck.

[2] Übersicht: W. Beck, B. Niemer, M. Wieser, Angew. Chem. 1993, 105, 969-996; Angew. Chem. Int. Ed. Engl. 1993, 32, 923-949.

[3] S. G. Davies, M. L. H. Green, D. M. P. Mingos, Nouv. J. Chim. 1977, 1, 445-447; S. G. Davies, M. L. H. Green, Tetrahedron 1978, 34, 3047-3077.

[4] R. E. Lehmann, J. K. Kochi, Organometallics 1991, 10, 190-202; J. K. Kochi, T. M. Bockman, Adv. Organomet. Chem. 1991, 33, 51-124; B. Olgemöller, W. Beck, Chem. Ber. 1981, $114,867-876$.

[5] S. C. Kao, P. P. Y. Lu, R. Pettit, Organometallics 1982, I, $911-918$.

[6] C. P. Casey, P. C. Vosejpka, Organometallics 1988, 7, 934-936; A. F. Dyke, S. A. R. Knox, M. J. Morris, P. J. Naish, J. Chem. Soc., Dalton Trans. 1983, 1417-1426.
[7] C. P. Casey, S. R. Marder, A. L. Rheingold, Organometallics 1985, 4, 762-766.

[8] C. P. Casey, M. S. Konings, S. R. Marder, Polyhedron 1988, 7, 881-902; C. P. Casey, M. S. Konings, S. R. Marder, J. Organomet. Chem. 1988, 345, 125-134.

[9] W. P. Schaefer, J. M. Spotts, S. R. Marder, Acta. Crystallogr., Sect. C, 1992, 48, 811-814.

[10] W. P. Fehlhammer, A. Schröder, J. Fuchs, E.-U. Würthwein, Angew. Chem. 1992, 104, 604-606; Angew. Chem. Int. Ed. Engl. 1992, 31, 590-592.

[11] K. Masuda, K. Nakano, T. Fukahori, H. Nagashima, K. Itoh, J. Organomet. Chem. 1992, 428, C21-C24.

[12] R. E. Dessy, R. L. Pohl, R. B. King, J. Am. Chem. Soc. 1966, $88,5121-5124$; C. K. Lai, W. G. Feighery, Y. Zhen, J. D. Atwood, Inorg. Chem. 1989, 28, 3929-3930.

[13] M. S. Corraine, C. K. Lai, Y. Zehn, M. R. Churchill, L. A. Buttrey, J. W. Ziller, J. D. Atwood, Organometallics 1992, 11, 35-40; W. S. Stiejewske, R. F. See, M. R. Churchill, J. D. Atwood, ibid. 1993, 12, 4413-4419, und dort zitierte Literatur.

${ }^{[14]}$ A. J. Birch, I. D. Jenkins, Transition Metal Organometallics in Organic Synthesis (Ed.: H. Alper), Academic Press, New York, 1976. Bd. 1; A. J. Pearson, Advances in Metallorganic Chemistry (Ed.: L. S. Liebeskind) Jai Press, London, 1989, Bd. 1; R. D. Pike, D. A. Sweigart, Synlett 1990, 565-571; G. R. Stephenson, P. W. Howard, D. A. Owen, A. J. Whitehead, J. Chem. Soc., Chem. Commun. 1991, 641-642; H. J. Knölker, R. Boese, K. Hartmann, Tetrahedron Lett. 1991, 17, 1953-1956; H. J. Knölker, M. Baumeister, J.-B. Pannek, Chem. Ber. 1992, 125, 2783-2793.

${ }^{[15]}$ F. O. Fischer, R. D. Fischer, Angew. Chem. 1960, 72, 919.

${ }^{[16]}$ P. Powell, Reactions of Coordinated Ligands (Ed.: P. S. Braterman), Plenum Press, New York, 1986, Bd. 1; P. L. Pauson, J. Organomet. Chem. 1980, 200, 207-221; A. J. Pearson, Comprehensive Organometallic Chemistry (Ed.: G. Wilkinson, F. G. A. Stone, E. W. Abel), Pergamon Press, Oxford, 1982, Bd. 8, S. 939-1011; L. A. P. Kane-Maguire, E. D. Honig, D. A. Sweigart, Chem. Rev. 1984, 84, 525-543; G. R. Stephenson, H Finch, D. Owen, S. Swanson, Tetrahedron 1993, 49, 5649-5662.

[17] W. Beck, B. Niemer, J. Breimair, J. Heidrich, J. Organomet. Chem. 1989, 372, 79-83. B. Niemer, J. Breimair, B. Wagner, K. Polborn, W. Beck, Chem. Ber. 1991, 124, 2227-2236.

${ }^{[18]}$ B. Niemer, J. Breimair, J. Heidrich, Chem. Ber. 1991, 124, $2237-2244$.

[19] B. F. G. Johnson, J. Lewis, T. W. Matheson, I. E. Ryder, M. V. Twigg, J. Chem. Soc., Chem. Commun. 1974, 269-270; B. F. G. Johnson, J. Lewis, I. E. Ryder, M. V. Twigg, J. Chem. Soc., Dalton Trans. 1976, 421-425; J. A. Smith, D. V. Howe, B. F. G. Johnson, J. Lewis, I. E. Ryder, J. Organomet. Chem. 1974, 82, $257-260$.

${ }^{[20]}$ A. Salzer, H. Werner, J. Organomet. Chem. 1975, 87, 101-108; A. Salzer, Inorg. Chim. Acta 1976, 17, 221-224.

[21] A. Salzer, P. Bigler, Inorg. Chim. Acta 1981, 48, 199-203.

${ }^{[22]} \mathrm{M}$. Wieser, K. Karaghiosoff, W. Beck, Chem. Ber. 1993, 126, $1081-1089$.

[23] G. E. Herberich, R. Michelbrink, Chem. Ber. 1970, 103, 3615-3618; Y.-H. Lai, W. Tam. K. P. C. Vollhardt, J. Organomet. Chem. 1981, 216, 97-103.

[24] W. E. Geiger, T. Gennett, G. A. Lane, A. Salzer, A. L. Rheingold, Organometallics 1986, 5, 1352-1359.

[25] C. P. Casey, M. S. Konings, M. A. Gohdes, M. W. Meszaros, Organometallics 1988, 7, 2103-2107.

[26] J. Breimair, M. Steimann, B. Wagner, W. Beck, Chem. Ber. 1990, 123, 7-10; M. Wieser, K. Sünkel, Ch. Robl, W. Beck, ibid. 1992, $125,1369-1373$.

[27] W. Beck, B. Niemer, B. Wagner, Angew. Chem. 1989, 101, 1699-1701; Angew. Chem. Int. Ed. Engl. 1989, 28, 1705-1706.

${ }^{[28]}$ H. J. Müller, U. Nagel, M. Steimann, K. Polborn, W. Beck, Chem. Ber. 1989, 122, 1387-1393.

[29] C. Krüger, Yi-Hung Tsay, Angew. Chem. 1971, 83, 250-251; Angew. Chem. Int. Ed. Engl. 1989, 10, 261-262; O. S. Mills, G. Robinson, Acta Crystallogr. 1963, 16, 758-761; M. R. Churchill, R. Mason, Proc. Chem. Soc. London 1967, 301, 433.

${ }^{[30]}$ R. B. King, F. G. A. Stone, Inorg. Synth. 1964, 7, 196-201.

[31] W. Hieber, G. Braun, Z. Naturforsch., Teil B, 1959, 14, 132-133; W. Hieber, G. Braun, W. Beck, Chem. Ber. 1960, 93, 901-908; W. Beck, K. Raab, Inorg. Synth. 1989, 26, 107.

${ }^{[32]}$ W. P. Fehlhammer, W. A. Herrmann, K. Öfele, Handbuch der Präparativen Anorganischen Chemie (Ed.: G. Brauer), Ferdinand Enke Verlag, Stuttgart, 1981, Bd. 3, S. 1919. 
[33] W. J. Carter, J. W. Kelland, S. J. Okrasinski, K. E. Warner, J. R. Norton, Inorg. Chem. 1982, 21, 3955-3960.

[34] Weitere Einzelheiten zur Kristallstrukturanalyse können beim Fachinformationszentrum Karlsruhe, Gesellschaft für wissenschaftlich-technische Information mbH, D-76344 EggensteinLeopoldshafen, unter Angabe der Hinterlegungsnummer CSD-
58208, der Autorennamen und des Zeitschriftenzitats angefordert werden. Zur Kristallstrukturanalyse von 4: Bei $2 \Theta$-Werten $>40^{\circ}$ zeigte der Kristall nur noch sehr geringes Beugungsvermögen. Große $U_{i-}$-Werte von $\mathrm{C} 2$ und $\mathrm{O} 2$.

${ }^{[35]}$ K. Jonas, Angew. Chem. Suppl. 1983, 1005-1006. 